\title{
Design of LPV-Based Sliding Mode Controller with Finite Time Convergence for a Morphing Aircraft
}

\author{
Nuan Wen, Zhenghua Liu, Yang Sun, and Lingpu Zhu \\ School of Automation Science and Electrical Engineering, Beihang University, Beijing 100191, China \\ Correspondence should be addressed to Nuan Wen; max_buaa3@163.com
}

Received 4 May 2017; Accepted 1 November 2017; Published 31 December 2017

Academic Editor: Kenneth M. Sobel

Copyright (C) 2017 Nuan Wen et al. This is an open access article distributed under the Creative Commons Attribution License, which permits unrestricted use, distribution, and reproduction in any medium, provided the original work is properly cited.

\begin{abstract}
This paper proposes a finite time convergence sliding mode control (FSMC) strategy based on linear parameter-varying (LPV) methodology for the stability control of a morphing aircraft subject to parameter uncertainties and external disturbances. Based on the Kane method, a longitudinal dynamic model of the morphing aircraft is built. Furthermore, the linearized LPV model of the aircraft in the wing transition process is obtained, whose scheduling parameters are wing sweep angle and wingspan. The FSMC scheme is developed into LPV systems by applying the previous results for linear time-invariant (LTI) systems. The sufficient condition in form of linear matrix inequality (LMI) constraints is derived for the existence of a reduced-order sliding mode, in which the dynamics can be ensured to keep robust stability and $L_{2}$ gain performance. The tensor-product (TP) model transformation approach can be directly applied to solve infinite LMIs belonging to the polynomial parameter-dependent LPV system. Then, by the parameter-dependent Lyapunov function stability analysis, the synthesized FSMC is proved to drive the LPV system trajectories toward the predefined switching surface with a finite time arrival. Comparative simulation results in the nonlinear model demonstrate the robustness and effectiveness of this approach.
\end{abstract}

\section{Introduction}

Morphing aircrafts, as a broad range of air vehicles and vehicle components that can make shape transition with internal morphing devices, can adapt to multimission requirements [1]. As a result, they will be a substitution, covering the roles of several different aircrafts that can allow optimized flight over a large flight envelope instead of merely one flight condition. However, this property will certainly bring great difficulties to the traditional modeling and control methods. Due to the massive change of aerodynamic configuration during flight, the morphing aircraft cannot be considered under full geometry variation and it must take the morphing structures as well as multirigid body variations into consideration. Therefore, unlike most conventional aircrafts which are only concentrated on a fixed-structure and treat themselves as a single rigid body, the consideration of morphing demands a combination with several research areas such as aerodynamic modeling [2], multirigid body dynamics [3], and flight control based on a large range of reference points [4]. Obviously, it is important to incorporate a wide range performance into modeling and controller for the dynamical systems that describe morphing aircrafts. LPV synthesis techniques naturally fit into this characteristic. By selecting appropriate operating conditions of the original nonlinear model, the complex dynamics in morphing process can be represented by LPV dynamic models [5]. LPV systems, being the specific instance of linear time-varying (LTV) systems, are the representation that the entries of the state-space matrices continuously depend on a time-varying parameter vector that belongs to a bounded compact set $[6,7]$. Using LPV techniques, the dynamics of the original nonlinear systems can be reduced to the linear equations. Meanwhile, the controller outputs will be continuously "scheduled" according to the system operating conditions. Comparing with the classical gain schedule techniques [8], LPV control can display prominent advantages as it can theoretically guarantee global stability and robustness over the whole operating envelope.

While its controller synthesis methods are theoretically well-founded based on LMI, LPV control is confronted with an inevitable challenge: direct generation of these methods to LPV systems will result in infinitely many LMI constraints 
that have to be satisfied [9]. There are three most common approaches to fixing this problem. An early possible way is to impose the constraints on a particular grid of the region of scheduled parameters [10]. But this discrete numerical method, which is similar to the classical gain schedule technique, will undoubtedly jeopardize robust stability and performance. Later developments in multiplier-based linear fractional transformation (LFT) synthesis make it possible to obtain finite-dimensional LMI constraints. In this case, the stability and performance are preserved, yet potential conservatism is introduced in the full-block S-procedure [11].The third approach to LPV controller synthesis is based on the properties of convex sets, in which it requires the LPV system to admit an affine or polytopic representation. Then, it is unnecessary to consider the full trajectories of scheduling parameter values, only the vertices of the convex hull need to satisfy the LMIs [12].However, a general LPV system may not be a desired polytopic or affine representation. Several kinds of mapping transformation are introduced to a model polytopic LPV system [13].The TP model transformation, as an equivalent transformation methodology in essence, is introduced in [14]. It can be directly employed to obtain a convex polytopic form with the known modeling accuracy, which greatly decreases the computing load of the controller design. Another key issue of the LPV system is to construct parameter-dependent Lyapunov functions to overcome potential conservatism brought by the slowly varying parameters. Aiming at providing the controller with less conservatism and better performance, $\mathrm{Wu}$ and Dong [11] incorporate quadratic LFT parameter-dependent Lyapunov functions and full-block multipliers into controller synthesis. Another way to construct Lyapunov functions with time-varying scheduled parameters is employing different Lyapunov functions at each of the vertices of the polytope LPV systems. By introducing slack variables, Peaucelle and Arzelier [15] separate the system matrices and the Lyapunov matrix to follow this method. Obviously, such LMI conditions will reduce conservatism at the expense of computational complexity.

For a nonlinear system of aircrafts, the Jacobian linearization approach is the most widespread methodology to obtain LPV models [5]. Generally, the designers built the global LPV model by interpolation and fitting of the LTI models which is formulated at different frozen points of interest throughout the operational envelope. Unfortunately, such steps inherently introduce uncertainties and model mismatches [16]. Moreover, the morphing transition will bring challenges for exact modeling of flight dynamics for its own sake. Both of these must be considered in the control design phase, which means that robust LPV control is attractive. Most of the current researches for LPV systems mainly focus on $H_{\infty}$ control techniques; these methods can reduce the impact of disturbance to some extent. However, unexpected uncertainties and model mismatches will deteriorate the performance of the controller [17]. Robust methods designed for uncertain LPV models have appeared in the literature. Considering actuator fault tolerant, a robust control strategy specified for LPV systems with polytopic uncertainty is proposed in [18]. For uncertain scheduling parameters appearing in LPV systems, the work in [19] develops a gain-scheduled output-feedback controller. In [20], a more general polytopic model describing LPV systems with state-dependent uncertain parameters is considered, and then the output-feedback controllers are designed by introducing the Lagrange multiplier term and adding some weight matrix variables. However, none of them consider general mismatched uncertainties appearing in LPV systems.

Sliding mode control is an attractive from 'uncertainty' standpoint, since the sliding surfaces are insensitive to matched or mismatched uncertainties [21]. To deal with uncertain systems with general mismatched uncertainties, a new reduced sliding mode is proposed in terms of LMI in [22]. Using the same method, the work in [23] ensures the robust stability and $L_{2}$ performance in the presence of an external disturbance. In [24], a robust adaptive sliding strategy based on model reference is presented for the tracking control problem of a special aircraft. However, all the methods cited above are based on LTI system descriptions of the plant and are restricted to the single operational condition. There are very limited researches on the use of sliding mode techniques for LPV systems. Most of them are restricted to sliding mode observers for a specific class of LPV systems $[25,26]$. By way of exception, the work in [27] proposes a time-varying sliding mode hyperplane for LPV systems. Also in [7], LPV-based integral sliding modes and control allocation are presented in a realistic operational environment. More recently, a standard secondorder sliding mode control approach is directly explored for the control of LPV systems in [28]; however, the approach for turning the infinite LMI constraints into a finite set of LMIs is simply a gridding-based synthesis without any theoretical proof.

Inspired by these works, the robust sliding mode control problem for uncertain LPV systems of a kind of large-scale morphing aircrafts is studied in this paper. First of all, according to multibody modeling and linearization, longitudinal nonlinear dynamic equations of the morphing aircraft in wing transition are simplified and transformed into an LPV model with mismatched uncertainties and external disturbance. Then, in the following LPV controller synthesis, we give a less conservative LMI existence condition of the reduced sliding dynamics. In order to avoid solving an infinite number of LMI constraints, the obtained LPV system is transformed into a convex polytopic by the TP model transformation. Finally, the synthesis of a parameterdependent reaching law is investigated for driving the system trajectories toward the predefined switching surface with a finite time arrival.

The exact contributions of this paper over previous works are concluded as follows:

(i) Compared with the works in $[4,17]$, we extend the morphing dimension into two degree of freedom: wingspan and sweep angle. Both detailed modeling process based on the Kane method, and explicit expressions of polynomial parameter-dependent LPV system are given. 
(ii) In this paper, we explore and evaluate the potential that the sliding mode control may have in the management of LPV systems with mismatched uncertainties. Unlike the controller in [28], which is just a simple mixture of the existed second-order sliding mode with fixed-structure for uncertain LPV systems, we adopt the reduced-order sliding mode controller to deal with uncertainties in LPV systems. Meantime, by applying the slack matrix method to derive a sufficient condition in the form of finite set of LMIs, we guarantee that the reduced-order dynamics in the sliding mode are robust and stable with the bounded $L_{2}$ gain performance. Then a parameter-dependent sliding mode control law can make the LPV system evolve into its sliding mode in a finite time.

The remainder of the paper is organized as follows: some preliminaries are given in Section 2. In Section 3, both a new morphing model and the corresponding LPV system are presented. In Section 4, the design process and stability analysis of FSMC law are demonstrated. In Section 5, numerical simulations are implemented to justify the performance of the proposed controller. The conclusion is shown in Section 6.

The notation is standard. The superscript " $T$ " denotes the transpose of a matrix. $\mathbf{P}>0(\mathbf{P} \geq 0)$ means that $\mathbf{P}$ is the real symmetric and positive-definite (semipositive definite); $\mathbf{A}<\mathbf{B}(\mathbf{A} \leq \mathbf{B})$ stands for that the matrix $\mathbf{A}-\mathbf{B}$ is the negative definite (semi-negative definite); I and $\mathbf{0}$ represent the identity matrix and a zero matrix, respectively; and $\|\bullet\|$ refers to the 2 norm for a vector or a matrix. In symmetric block matrices, we use $*$ to represent a term that is induced by symmetry. diag represents a diagonal matrix. Both $a^{\prime}$ and $\dot{a}$ denote the first order derivative. $a^{\prime \prime}$ denotes the second-order derivative. $\times$ denotes the outer product of vectors.

\section{Preliminary Results}

Lemma 1. (see [23]). The following block matrix inequality holds

$$
\left[\begin{array}{ll}
A_{11} & A_{12} \\
A_{12}^{T} & A_{22}
\end{array}\right] \leq\left[\begin{array}{ll}
\tilde{A}_{11} & A_{12} \\
A_{12}^{T} & A_{22}
\end{array}\right],
$$

if $A_{11} \leq \tilde{A}_{11}$ holds.

Lemma 2 (see [24]). $\mathbf{X}$ and $\mathbf{Y}$ are real matrices (or vectors) of appropriate dimensions; for any scalar $\varepsilon>0$, one has

$$
\mathbf{X}^{T} \mathbf{Y}+\mathbf{Y}^{T} \mathbf{X} \leq \varepsilon \mathbf{X}^{T} \mathbf{X}+\varepsilon^{-1} \mathbf{Y}^{T} \mathbf{Y}
$$

Lemma 3 (see [12]). The following statements are equivalent:

$$
\begin{array}{r}
{\left[\begin{array}{c}
I \\
N
\end{array}\right]^{T} M\left[\begin{array}{c}
I \\
N
\end{array}\right]<0,} \\
M+\left[\begin{array}{c}
N^{T} \\
-I
\end{array}\right] G^{T}+G[N-I]<0 .
\end{array}
$$

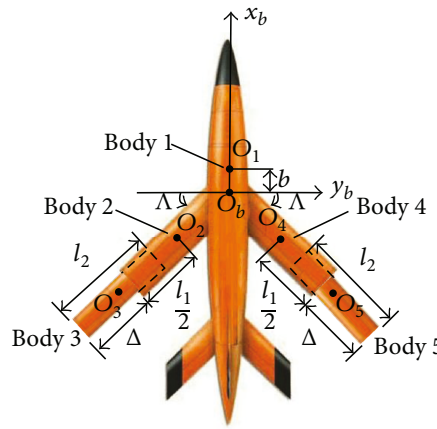

Body 1: fuselage

Body 2, Body 4: fixed wings

Body 3, Body 5: telescopic wings

(a)

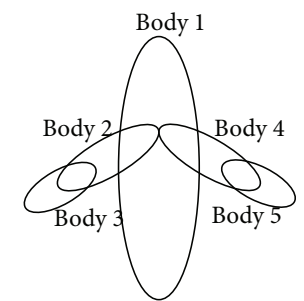

Body 1: fuselage Body 2, Body 4: fixed wings Body 3, Body 5: telescopic wings

(b)
FIgURE 1: (a) The Morphing aircraft with variable sweep angle and variable span. (b) Depiction of the multibody representation.

Lemma 4 (see [29]). Assume that a continuous, nonnegative function $V(t)$ satisfies the differential inequality $\dot{V}(t) \leq-\alpha$ $V^{\eta}(t)$, where $\alpha>0$ and $0<\eta<1$ are constants. Then, for any given $t_{0}$

$$
V^{1-\eta}(t) \leq V^{1-\eta}\left(t_{0}\right)-\alpha(1-\eta)\left(t-t_{0}\right), \quad t_{0} \leq t \leq t_{r}
$$

and $V(t)=0$ and $t \geq t_{r}$ with $t_{r}$ given by

$$
t_{r}=t_{0}+\frac{V^{1-\eta}\left(t_{0}\right)}{\alpha(1-\eta)}
$$

\section{Model and Problem Formulation}

Flight dynamics of a morphing aircraft is a key component in the design process and must therefore be modeled accurately as well as practically. In this section, the longitudinal nonlinear dynamic equations of the morphing aircraft are formulated first, and then the linear parameter-dependent model is developed for controller synthesis.

3.1. Longitudinal Nonlinear Dynamic Modeling for the Morphing Aircraft. While numerous morphing designs exist for advanced aircrafts, we consider a new type of large-scale morphing aircrafts [30] depicted in Figure 1(a), whose wings can extend, contract, and rotate to a certain extent.

The Teledyne Ryan BQM-34 "Firebee", which is designed as a high-speed target drone, is chosen as the baseline platform [31]. The aircraft is equipped with an easily removable wing that could be modified into a morphing wing. Also, many variants of this aircraft have been constructed to accommodate mission requirements such as subsonic or supersonic flight, reconnaissance, and combat. Next, we suppose that the wing of each side of the aircraft is equipped with an inner wing and an outer wing. In wing morphing process, the inner can 
TABLE 1

\begin{tabular}{lc}
\hline$b$ & $0.3 \mathrm{~m}$ \\
$\Lambda$ & $0 \sim 45 \mathrm{deg}$ \\
$\Delta$ & $0 \sim 2 \mathrm{~m}$ \\
$J_{y}$ & $3107.53 \mathrm{~kg} \cdot \mathrm{m}^{2}$ \\
$l_{1}$ & $2.5 \mathrm{~m}$ \\
$l_{2}$ & $2.0 \mathrm{~m}$ \\
$m_{b}$ & $771.79 \mathrm{~kg}$ \\
$m_{1}$ & $54 \mathrm{~kg}$ \\
$m_{2}$ & $13.605 \mathrm{~kg}$ \\
$T_{\delta_{T}}$ & $129.27 \mathrm{~N} / \%$ \\
\hline
\end{tabular}

rotate with $O_{b}$ and the outer will keep level and move linearly along with the airfoil (see Figure 1(a)). Owing to this specific structure, the morphing aircraft can change the wing sweep angle and wingspan to accommodate mission requirements. The maximum wing sweep angle $\left(\Lambda_{\max }\right)$ is $45^{\circ}$ and the maximum extension $\left(\Lambda_{\max }\right)$ is $2 \mathrm{~m}$. The geometry of the other parts (fuselage, vertical tail, horizontal tail, etc.) remains unchanged in this paper.

To simplify the modeling process, we make the following assumption:

Assumption 1. In the morphing process, the left wing and the right wing will realize the synchronous motion. The symmetry axis of the fuselage lies in the same plane as the wings on both sides.

The traditional approach to modeling a conventional aircraft is to treat the whole plane as a single rigid body without regard for the complications brought by the aeroelastic problems. The morphing aircraft with large-scale varations mentioned above, however, cannot generally be modeled as a single rigid body due to the varying inertial properties caused by the changes of geometric parameters of the wings. To describe the aircraft dynamic behavior accurately, the whole structural changes of each part must be taken into account properly, which means that multibody modeling is an appropriate solution. The Kane method is a simple and efficient approach to derive the dynamic model of a multibody system [32]. Thereby, the longitudinal dynamics of the morphing motion is described by using Kane method in this paper.

The aircraft under consideration consists of five separate rigid bodies (see Figure 1(b)): fuselage (body 1), inner part of left wing (body 2), outer part of left wing (body 3 ), inner part of right wing (body 4), and outer part of right wing (body 5), and their masses are $m_{b}, m_{1}, m_{2}, m_{1}, m_{2}$, respectively. The important parameters of the morphing aircraft are given in Table 1.

Firstly, as shown in Figure 2, we set the origin of the whole aircraft body coordinate frame $O_{b} x_{b} y_{b} z_{b}$ to locate at the center the wing (the unit vectors corresponding to the axes $x_{b}, y_{b}$ and $z_{b}$ are $\mathbf{e}_{\mathbf{x}}^{\mathbf{b}}, \mathbf{e}_{\mathbf{y}}^{\mathbf{b}}$ and $\mathbf{e}_{\mathbf{z}}^{\mathbf{b}}$ ), and the ground

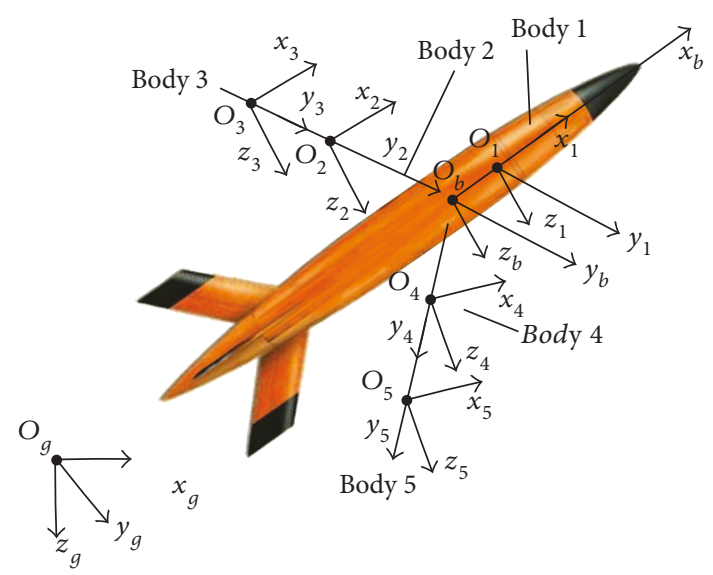

Figure 2: Coordinate axis system.

coordinate system is described as $O_{g} x_{g} y_{g} z_{g}$. The coordinate frames of morphing aircraft's fuselage and the other several moving parts of the wing are described as $O_{i} x_{i} y_{i} z_{i}(i=1, \ldots$ $, 5)$, taking the center of mass of each body as the origin correspondingly. In what follows, Kane's equations will be established in the reference frame $O_{b} x_{b} y_{b} z_{b}$.

Assumption 2. Both the aerodynamic force and the thrust are merely acting on the fuselage (body 1). And the other parts suffer the effects of gravity only.

There are four steps to establish the Kane equations, as depicted in Figure 3. Here, we only consider the longitudinal responses in morphing process. Thus, $(u, w, q)$ are selected as independent generalized speed variables.

In Figure $3, F_{O_{b}}^{(u)}, F_{O_{b}}^{(w)}$ and $F_{O_{b}}^{(q)}$ are generalized active forces corresponding to $(u, w, q) \cdot \mathbf{F}_{O_{b}}^{i}$ and $\mathbf{M}_{O_{b}}^{i}$ are generalized active forces and active moments of the ith body projected into $O_{b} x_{b} y_{b} z_{b}$. $\tilde{F}_{O_{b}}^{(u)}, \tilde{F}_{O_{b}}^{(w)}$, and $\tilde{F}_{O_{b}}^{(q)}$ are generalized inertial forces relative to $(u, w, q) . \tilde{\mathbf{F}}_{O_{b}}^{i}$ and $\tilde{\mathbf{M}}_{O_{b}}^{i}$ stand for the generalized inertial force and inertial moment of the $i$ th body. $\mathbf{U}_{O_{b}}^{i(\sim)}$ and $\mathbf{W}_{O_{b}}^{i(\sim)}$ are partial velocities of the $i$ th body located into $O_{b} x_{b} y_{b} z_{b}$. The specific definitions of these variables are given in Appendix A.

According to the fourth step in Figure 3, the detailed expressions of the Kane equations of longitudinal dynamics of the morphing aircraft can be written as follows:

$$
\begin{aligned}
f_{1}\left(\dot{u}, w, q, \alpha, \theta, \Lambda, \Lambda^{\prime}, \Lambda^{\prime \prime}, \Delta^{\prime}, \Delta^{\prime \prime}\right) & =0, \\
f_{2}\left(\dot{w}, \dot{q}, q, u, \alpha, \theta, \Lambda, \Lambda^{\prime}, \Delta^{\prime}\right) & =0, \\
f_{3}\left(\dot{w}, \dot{q}, q, u, \alpha, \theta, \Lambda, \Lambda^{\prime}, \Delta^{\prime}\right) & =0 .
\end{aligned}
$$

The explicit expressions of (6) are given in Appendix B.

By introducing (7), (6) can be transformed into a wind coordinate frame. Considering the kinematic 


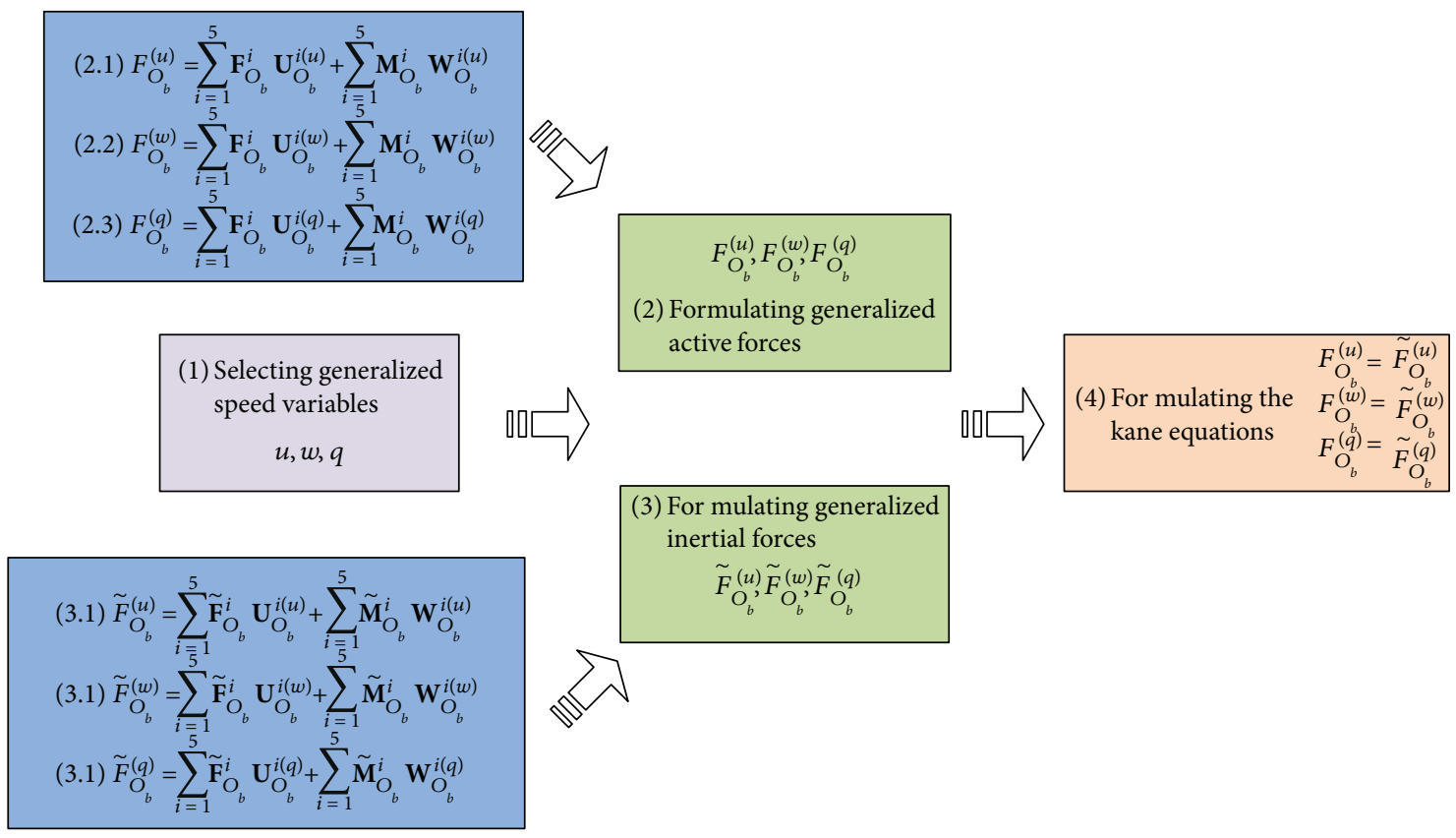

FIGURE 3: Kane modeling procedure.

equation (8), we finally get the full longitudinal dynamic equation (9).

$$
\begin{aligned}
u & =V \cos \alpha, \\
\dot{u} & =\dot{V} \cos \alpha-V \sin \alpha \dot{\alpha}, \\
w & =V \sin \alpha, \\
\dot{w} & =\dot{V} \sin \alpha+V \cos \alpha \dot{\alpha}, \\
\dot{\theta} & =q, \\
\dot{h} & =V \sin (\theta-\alpha), \\
\mathbf{E}\left[\begin{array}{c}
\dot{V} \\
\dot{\alpha} \\
\dot{q} \\
\dot{\theta} \\
\dot{h}
\end{array}\right] & =\left[\begin{array}{c}
F_{1} \\
F_{2} \\
F_{3} \\
q \\
V \sin (\theta-\alpha)
\end{array}\right]+\left[\begin{array}{c}
W_{1} \\
W_{2} \\
W_{3} \\
0 \\
0
\end{array}\right] .
\end{aligned}
$$

The explicit expressions of $\mathbf{E}, F_{1}, F_{2}, F_{3}, W_{1}, W_{2}$, and $W_{3}$ are given in Appendix C.

Remark 1. By adopting the Kane modeling, only the position, velocity and acceleration of $\Lambda$ and $\Delta$ appear in the dynamic modeling equation (9). They can be computed and manipulated more practically than the position, velocity, and acceleration of the center of mass or instantaneous inertia tensor and its derivative [17]. This shows another major superiority of the Kane method. Notice that we will apply our designed LPV-based controller on the original nonlinear equation (9), and the exact values of $\Lambda^{\prime}, \Lambda^{\prime \prime}$ and $\Delta^{\prime}, \Delta^{\prime \prime}$ at every moment must be obtained so that we can substitute them into (9). Therefore, the motion trajectories of sweep angle and span need to be scheduled appropriately in advance. We will discuss it in the following section.

Remark 2. $W_{1}, W_{2}$, and $W_{3}$ including the first and the second-order derivative of $\Lambda$ and $\Delta$ are the force and moment variations affected by the morphing movement, which can be considered as the additional disturbances in the wing morphing dynamic response [17]. However, in the following section, these extra forces and moments are proved to have such slight influence to the entire dynamic equations that they can be ignored in the following linearization procedure.

3.2. Longitudinal LPV Model for the Morphing Aircraft. In this paper, the Jacobian linearization approach is adopted to transform the nonlinear model of the morphing aircraft into an LPV model. The flight condition of interest is selected as the altitude $h_{0}=9144 \mathrm{~m}$ and the Mach number $\mathrm{Ma}=0.5$. By defining the scheduling variables $\lambda=\Lambda / 45^{\circ}$ and $\xi=\Delta / l_{1}$, we get $\lambda \in[0,1]$ and $\xi \in[0,0.8]$.

Assumption 3 (see [17]). In this paper, unsteady aerodynamic effects are neglected. We only focus on the aerodynamic forces and moments introduced by the quasi-steady assumption; that is, the aerodynamic forces and moments of the morphing process in different wing configurations are nearly identical to those of the corresponding static configuration. 


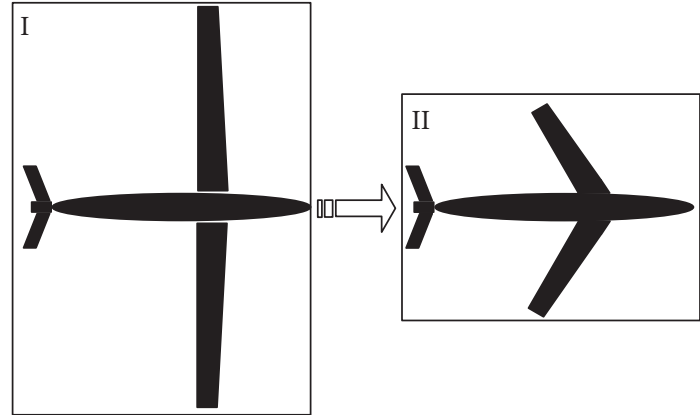

Figure 4: Morphing process.

The lift, drag, pitching moments, and thrust in (9) are given as

$$
\begin{aligned}
D & =\frac{1}{2} \rho V^{2} S_{\mathrm{w}} C_{D_{\alpha}}(\alpha), \\
L & =\frac{1}{2} \rho V^{2} S_{\mathrm{w}} C_{L}=\frac{1}{2} \rho V^{2} S_{\mathrm{w}}\left(C_{L_{\alpha=0}}+C_{L_{\alpha}} \alpha+C_{L_{\delta e}} \delta_{e}\right), \\
M & =\frac{1}{2} \rho V^{2} S_{\mathrm{w}} \bar{c} C_{M} \\
& =\frac{1}{2} \rho V^{2} S_{\mathrm{w}} \bar{c}\left(C_{M_{\alpha=0}}+C_{M_{\alpha}} \alpha+C_{M_{\delta e}} \delta_{e}+\frac{\bar{c}}{2 V} C_{m_{q}} q\right), \\
T & =T_{\delta_{\mathrm{t}}} \delta_{\mathrm{t}} .
\end{aligned}
$$

Choosing $\lambda=0,0.2,0.4, \ldots, 1.0$ and $\xi=0,0.2,0.4, \ldots$, 0.8 as the reference points, then the corresponding aerodynamic parameters for these configurations can be approximately calculated through computational fluid dynamics (CFD). Firstly, the outline dimensions of the aircraft are obtained by observation. Then the three-dimensional model of the morphing aircraft under different configurations is established by Catia. After gridding model and importing it into the fluent software of ANSYS, the aircraft aerodynamic force and moment can be calculated.

Finally, we integrate, analyze, and calculate the obtained data of those static configurations and the expressions of aerodynamic derivatives of the morphing aircraft during the whole morphing process can be derived by least square fitting of MATLAB. Therefore, we get the continuous aerodynamic parameter expressions in Appendix C.

Next, we take the following morphing process (shown in Figure 4) as an example. Configuration I stands for loiter mode with $\lambda=0$ and $\xi=0.8$. With this configuration, the aircraft is provided with a larger lift-to-drag ratio that can reduce energy consumption during loiter. Configuration II represents dash mode with $\lambda=1$ and $\xi=0$, and the aircraft can obtain more speed with smaller drag force.

In Figure 5, where the values of aerodynamic coefficients are varying along with an angle of attack from configuration I to configuration II, we can conclude that the aerodynamic coefficients of the morphing aircraft change greatly during the morphing process. For example, the lift coefficient decreases by about 53\% with $\alpha=5^{\circ}$ when the aircraft changes from configuration I to configuration II. The drag coefficient has little changes in the range of the small angle of attack. Furthermore, the lift-to-drag ratio varies greatly under different aerodynamic configurations, which provides the possibility for the morphing aircraft to complete different tasks. The pitching moment coefficient has a dramatic change during the morphing process, which is caused by the difference of the distance between the aerodynamic center and the mass center.

After linearizing the morphing model equation (9) at each equilibrium reference point, the longitudinaldirectional dynamics can be expressed as

$$
\dot{\mathbf{x}}=\mathbf{A}(\lambda, \xi) \mathbf{x}+\mathbf{B}_{2}(\lambda, \xi) \boldsymbol{u}
$$

where $\mathbf{x}=[\Delta V, \Delta \alpha, \Delta q, \Delta \theta, \Delta h]^{T}$ and $\mathbf{u}=\left[\Delta \delta_{e}, \Delta \delta_{t}\right]^{T}$.

It is easy to verify that the elements in the state-space matrices depend on the scheduling variables, and hence we get

$$
\begin{aligned}
\mathbf{A}(\lambda, \xi) & =\left[\begin{array}{ccccc}
A_{11}(\lambda, \xi) & A_{12}(\lambda, \xi) & 0 & -g & 0 \\
0 & A_{22}(\lambda, \xi) & 1 & 0 & 0 \\
0 & A_{32}(\lambda, \xi) & 0 & 0 & 0 \\
0 & 0 & 1 & 0 & 0 \\
0 & -V_{0} & 0 & V_{0} & 0
\end{array}\right], \\
\mathbf{B}_{2}(\lambda, \xi) & =\left[\begin{array}{cc}
B_{11}(\lambda, \xi) & 0.1425 \\
B_{21}(\lambda, \xi) & 0 \\
B_{31}(\lambda, \xi) & 0 \\
0 & 0 \\
0 & 0
\end{array}\right] .
\end{aligned}
$$

The explicit expressions of the elements in the above matrixes are given in the Appendix D.

Notice that the LPV model equation (11) is constructed on a family of LTI plants linearized with respect to a set of equilibrium points of a nonlinear system that represents the flight envelope of interest. Therefore, the open-loop dynamic response of morphing process produced by these two models should be similar, which guarantees that LPV model can be a reliable alternative to describe the original nonlinear system (9).

However, considering the existence of $\Lambda^{\prime}, \Lambda^{\prime \prime}, \Delta^{\prime}$, and $\Delta^{\prime \prime}$ in a nonlinear system (6), the motion trajectories of the sweep angle and span need to be scheduled. According to the work in [2], we know that the variation rate of the morphing movement will not change the trend of dynamic responses, but it will have a marked impact on the pace of change of the dynamic responses. Taking the long-period mode $V$ and the short-period mode $q$ as examples, Figure 6 shows that the dynamic responses vary along with span and sweep angle in different morphing velocities. Also, we must notice that too much fast variation rate of morphing movement will definitely bring a nonignorable unsteady aerodynamic effect. 

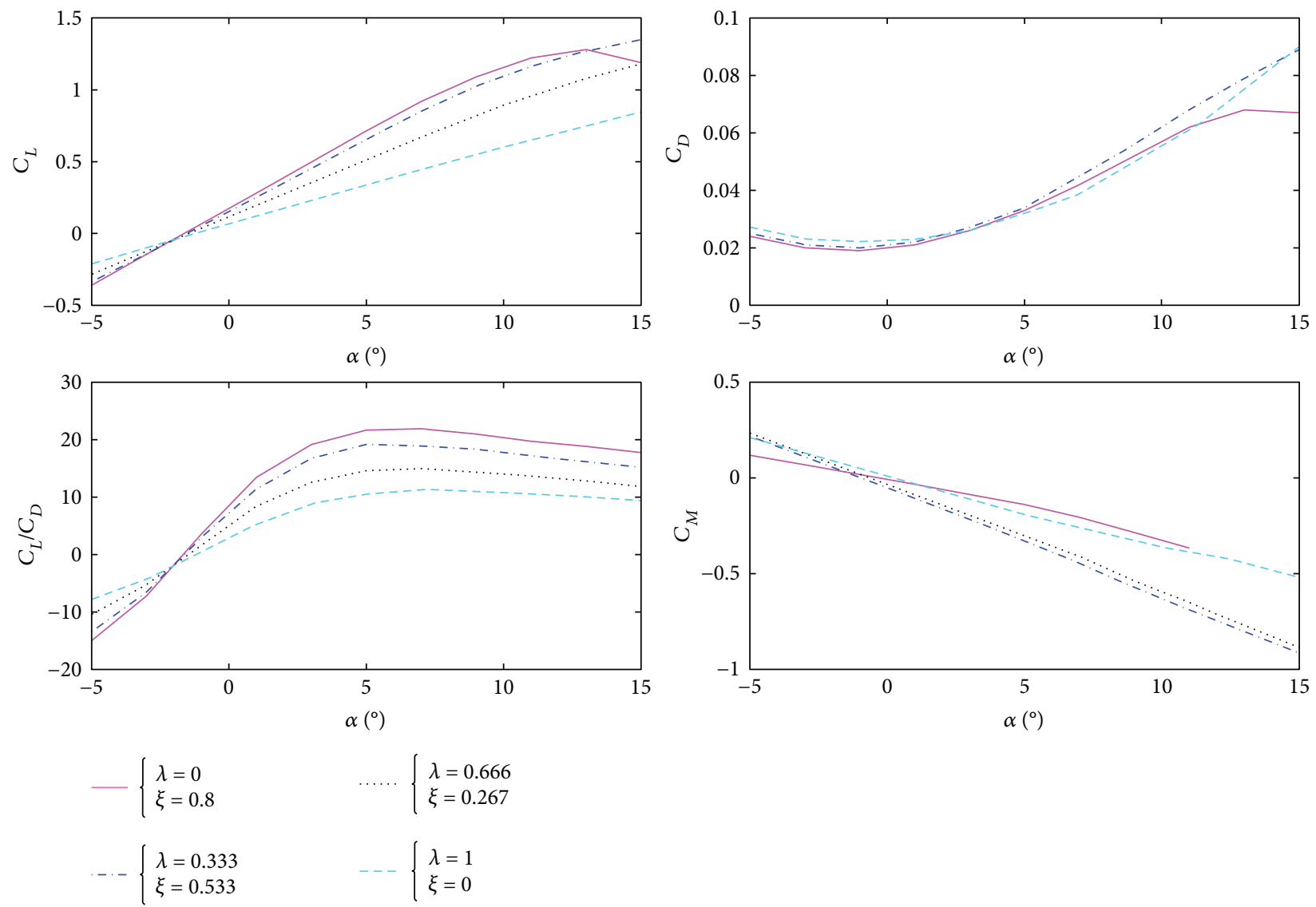

FIgURe 5: Aerodynamic coefficients.

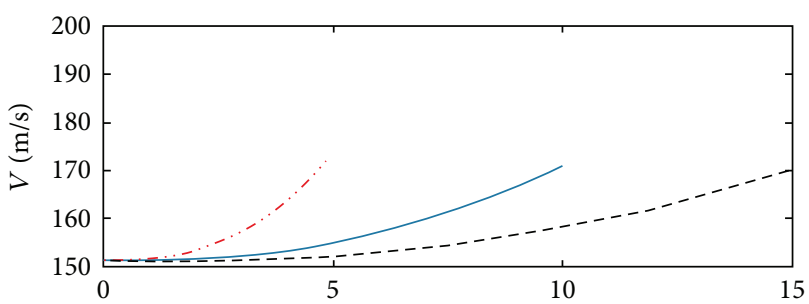

arrange morphing mechanism motions. The servomotor system of the morphing mechanism is subject to acceleration and speed constraints. The trajectory generation is formulated as a linear-constrained optimal control problem and the Pontryagin's maximum principle can be applied to derive necessary optimality conditions. Instead of solving multipoint boundary value problems directly, we can solve this constrained optimal control by transforming it into an optimal time interval of the speed-constrained arc and a specific acceleration-constrained optimal control problem, which can be rapidly solved using the numerical method and analytic method. Here, the energy-optimal morphing trajectory is presented without concrete derivation and the details are given in [33]. The proposed trajectory not only can guarantee the smooth morphing movement but also will save the additional energy caused by morphing mechanisms.

Here we define

$$
\begin{aligned}
& \Lambda_{\max }^{\prime}=7(\mathrm{deg} / \mathrm{s}), \\
& \Lambda_{\max }^{\prime \prime}=2\left(\mathrm{deg} / \mathrm{s}^{2}\right), \\
& \Lambda_{\min }^{\prime \prime}=-2\left(\mathrm{deg} / \mathrm{s}^{2}\right), \\
& \Delta_{\max }^{\prime}=0.31(\mathrm{~m} / \mathrm{s}), \\
& \Delta_{\max }^{\prime \prime}=0.09\left(\mathrm{~m} / \mathrm{s}^{2}\right), \\
& \Delta_{\min }^{\prime \prime}=-0.09\left(\mathrm{~m} / \mathrm{s}^{2}\right) .
\end{aligned}
$$
driven by servo actuators, then a real-time energy-optimal trajectory generation for a servomotor system is adopted to 

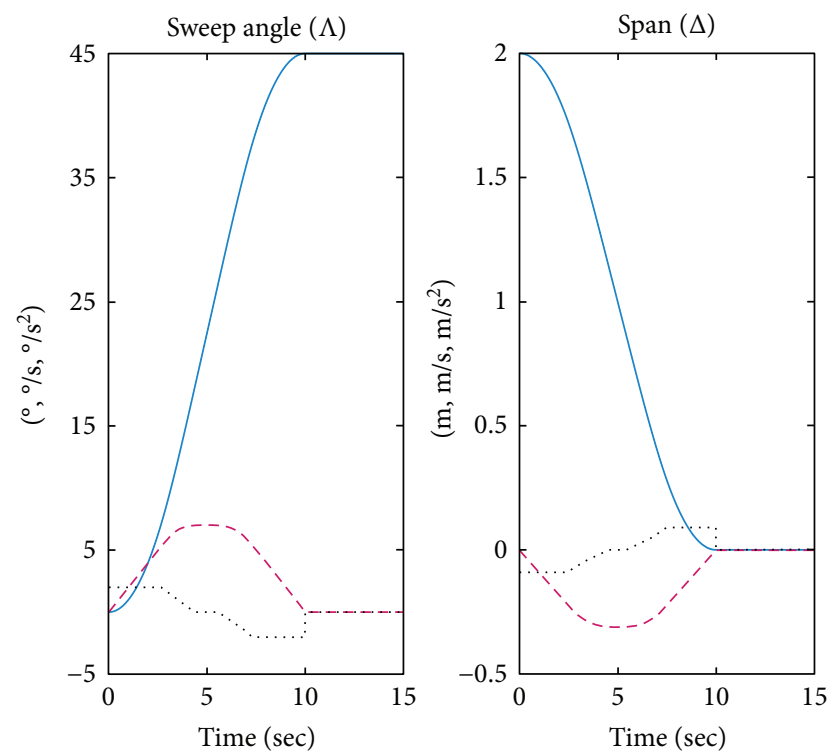

$$
\begin{aligned}
& -\Lambda \\
& ---\Lambda^{\prime} \\
& \cdots \cdots \cdot \Lambda^{\prime \prime}
\end{aligned}
$$$$
\begin{aligned}
& -\Lambda \\
& ---\Lambda^{\prime} \\
& \cdots \cdots \Lambda^{\prime \prime}
\end{aligned}
$$

Figure 7: Energy-optimal trajectories.

Then, the energy-optimal morphing trajectories from configuration I to configuration II are designed and shown in Figure 7.

The distinct responses of the two kinds of models are shown in Figure 8. We can see that the altitude gradually decreases and at the same time the speed increases accordingly. This is mainly due to the reduction of the wing area brought by the contraction and sweep motion of the wing. The value of lift coefficient is becoming smaller along with a reduction of the wing area. Therefore, the lift force belonging to the vertical direction is not large enough to balance the gravity, which provides the aircraft with the downward acceleration. Meantime, with the decrease of pitch angle, the component of the engine thrust has changed from the upward direction into the downward direction. And this will certainly result in the downward acceleration. During the overall morphing process, the angle of attack increases as the value of the vertical velocity component becomes larger. At the end of the morphing process, the aircraft is going to seek a new balance state where the speed and the angle of attack are larger to counteract the smaller value of lift force brought by the reduction of the wing area. Furthermore, the discrepancies of dynamic responses between the LPV model and the nonlinear model are small. The LPV model is able to capture the dynamic behavior and match the nonlinear model to some extent. Therefore, the validated LPV model can become an object for the controller synthesis.

Figure 9 also shows the proportion of the additional disturbances $W_{1}, W_{2}$, and $W_{3}$ in the whole dynamic model. It is easy to see that $W_{1}, W_{2}$, and $W_{3}$ are not in the same order magnitude comparing with $F_{1}, F_{2}$, and $F_{3}$. Therefore, we can simplify the linearization procedure by omitting these extra forces and moments. However, this will
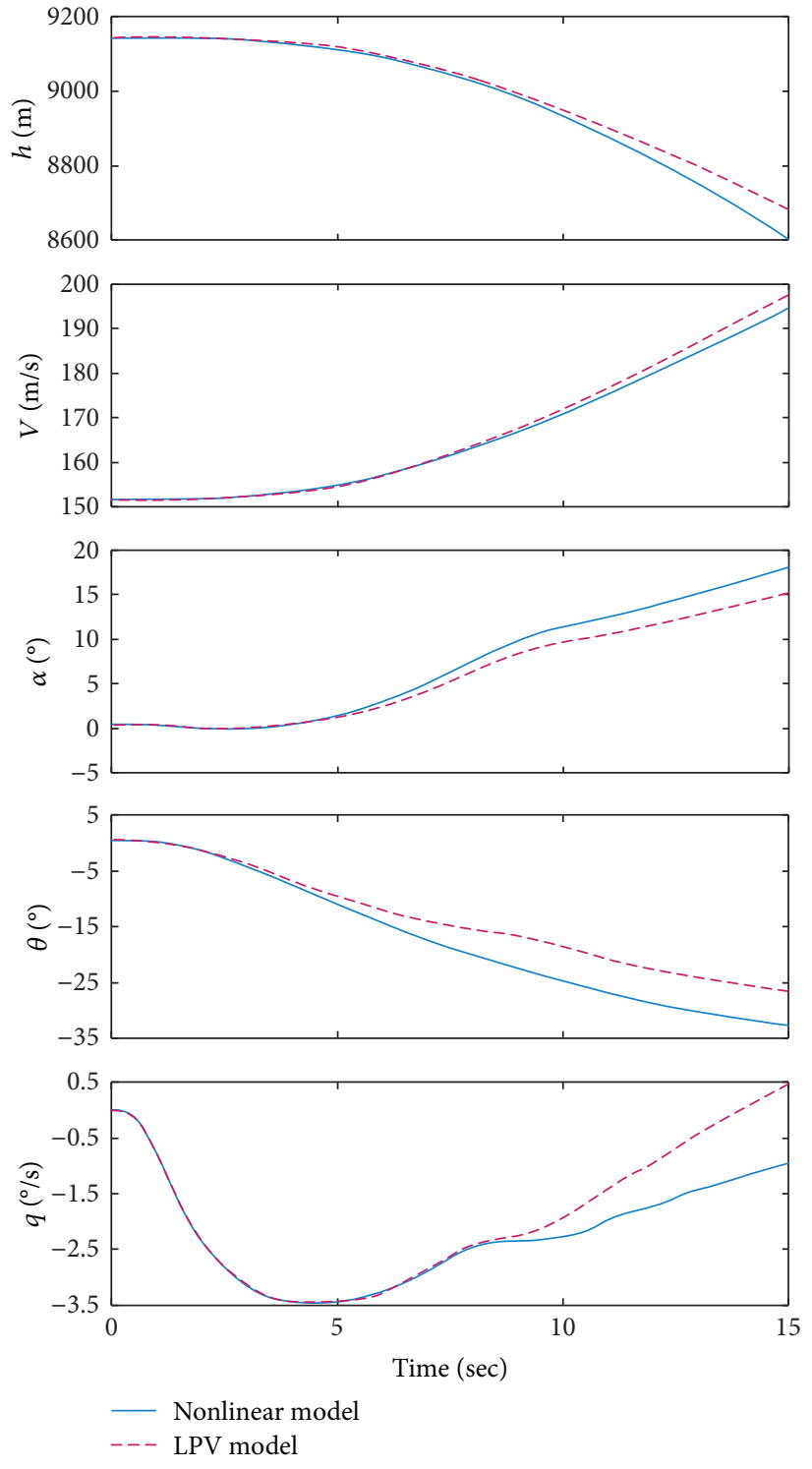

FIGURE 8: Nonlinear model versus the LPV model.

certainly propose a higher requirement for the robustness of the controller.

Moreover, the aerodynamics in wing morphing obtained by CFD is regarded as being quasi steady. Unfortunately, the unsteady aerodynamic characteristic caused by dynamic hysteresis effect does exist in the practical morphing process [34]. This will bring modeling uncertainties of the aerodynamics in the nonlinear model to a certain extent. Meanwhile, external disturbances such as gust wind or turbulent interference exert considerable influence during flight. Therefore, now we consider the more challenging case in which the LPV system is affected by parametric perturbation in the system matrices and external disturbance, that is,

$$
\dot{\mathbf{x}}=[\mathbf{A}(\lambda, \xi)+\Delta \mathbf{A}(\lambda, \xi)] \mathbf{x}+\mathbf{B}_{1} \mathbf{w}+\left[\mathbf{B}_{2}(\lambda, \xi)+\Delta \mathbf{B}_{2}(\lambda, \xi)\right] \mathbf{u} .
$$



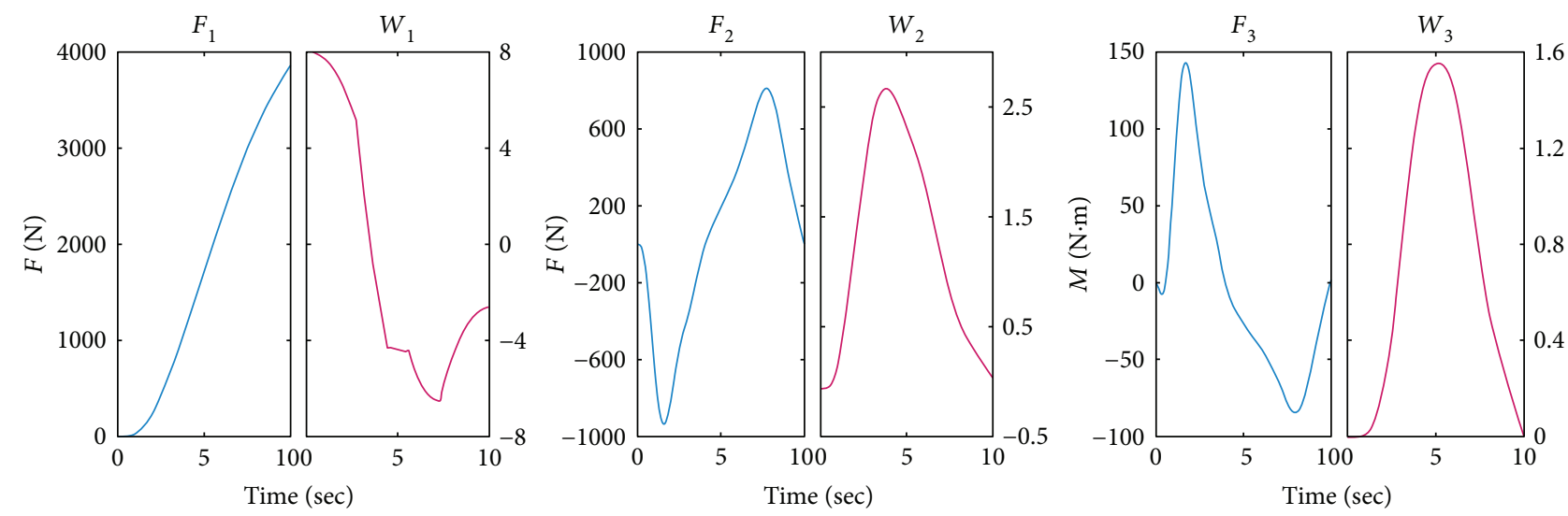

Figure 9: Conventional dynamic versus additional dynamic.

In view of this, robust control techniques for uncertain LPV system are indispensable to maintain nominal performance and robustness in the face of massive uncertain factors.

\section{Sliding Mode LPV Control}

In order to guarantee the stability of the morphing aircraft, especially the stability during the morphing process, an appropriate controller has to be designed to achieve the following objectives:

(i) Not only at any static morphing completion state but also during the dynamic morphing process, the closed loop of the flight system should stable globally.

(ii) Keep the altitude and speed constant during the transition from configuration I to configuration II.

To accomplish a smooth transition process against a great deal of uncertainty, we design the FSMC for the uncertain LPV system (14).

4.1. Sliding Mode Dynamics Analysis. Consider the system with the following state-space representation for the LPV system

$$
\begin{aligned}
\dot{\mathbf{x}}(t) & =(\mathbf{A}(\Theta)+\Delta \mathbf{A}(\Theta)) \mathbf{x}(t)+\mathbf{B}_{1}(\Theta) \mathbf{w}(t)+\mathbf{B}_{2} \mathbf{u}(t), \\
\mathbf{z}(t) & =\mathbf{C}_{1}(\Theta) \mathbf{x}(t)+\mathbf{D}_{1}(\Theta) \mathbf{w}(t),
\end{aligned}
$$

where $\mathbf{x} \in R^{n_{\mathbf{x}}}$ is the state, $\mathbf{w} \in R^{n_{\mathbf{w}}}$ is the external disturbance, $\mathbf{u} \in R^{n_{\mathrm{u}}}$ is the control input, and $\mathbf{z} \in R^{n_{\mathbf{z}}}$ is the performance of the system. We denote continuous, measurable quantities that range in some admissible set $\Theta$ as scheduling variables, $\Theta(t)=\left[\theta_{1}(t), \theta_{2}(t), \ldots, \theta_{n_{\theta}}(t)\right]^{T} . \Delta \mathbf{A}(\Theta)$ represents the system matrix uncertainty.

The following assumptions are made.

Assumption 4. The pair $\left(\mathbf{A}(\Theta), \mathbf{B}_{2}\right)$ is stabilizable for all $\Theta$, and the state $\mathbf{x}$ is available.

Assumption 5. The input matrix $\mathbf{B}_{2}$ has full column rank and $\mathbf{B}_{\perp}$ is any basis of the null space of $\mathbf{B}_{2}^{T}$.
Assumption 6. There exists a known constant $\varepsilon_{\Delta}$ such that for all $\Theta,\|\Delta \mathbf{A}(\Theta)\| \leq \varepsilon_{\Delta}$. As for $\mathbf{w}(t)$, we set $\varepsilon_{w}=\|\mathbf{w}(t)\|_{\max }$ is the upper bound of $\|\mathbf{w}(t)\|$ which is assumed to be obtained in advance.

Remark 3. The matrix $\mathbf{B}_{2}$ is assumed to be constant. When this is not the case, low-pass filters with sufficiently large bandwidth can be used to filter the system's inputs and hence to move all the time-varying parameters into the state matrix [12]. Therefore, even in the case when $\mathbf{B}_{2}$ is the functions of $\Theta$, the augmented model of the system can be converted into the form (15). In consideration of the system (14), we define a new control input $\tilde{\mathbf{u}}$ by

$$
\begin{aligned}
\dot{\mathbf{x}}_{\mathbf{u}} & =\mathbf{A}_{\mathbf{u}} \mathbf{x}_{\mathbf{u}}+\mathbf{B}_{\mathbf{u}} \tilde{\mathbf{u}}, \\
\mathbf{u} & =\mathbf{C}_{\mathbf{u}} \mathbf{x}_{\mathbf{u}},
\end{aligned}
$$

where $\mathbf{A}_{\mathbf{u}}$ is stable. Then the augmented LPV plant is described by

$$
\begin{aligned}
{\left[\begin{array}{c}
\dot{\mathbf{x}} \\
\dot{\mathbf{x}}_{\mathbf{u}}
\end{array}\right]=} & {\left[\begin{array}{cc}
\mathbf{A}(\lambda, \xi)+\Delta \mathbf{A}(\lambda, \xi) & {\left[\mathbf{B}_{2}(\lambda, \xi)+\Delta \mathbf{B}_{2}(\lambda, \xi)\right] \mathbf{C}_{\mathbf{u}}} \\
0 & \mathbf{A}_{\mathbf{u}}
\end{array}\right] } \\
& \cdot\left[\begin{array}{c}
\mathbf{x} \\
\mathbf{x}_{\mathbf{u}}
\end{array}\right]+\left[\begin{array}{c}
\mathbf{B}_{1} \\
0
\end{array}\right] \mathbf{w}+\left[\begin{array}{c}
0 \\
\mathbf{B}_{\mathbf{u}}
\end{array}\right] \tilde{\mathbf{u} .}
\end{aligned}
$$

For now, note that the control matrix $\mathbf{B}_{2}$ is now parameterfree. The bandwidth of filter of (16) must be chosen larger than the desired system bandwidth. With this constraint, the proposed prefiltering will not significantly alter the original system (14). Therefore, the specific assumption of $\mathbf{B}_{2}$ in the system (15) is withheld.

Remark 4. As described in Assumption 5, the uncertainty $\Delta \mathbf{A}(\Theta)$ is not necessary to satisfy the matched conditions. To stabilize the LPV system, the FSMC technique is utilized here. First, a sliding mode surface function is designed as

$$
s(t)=\mathbf{B}_{2}^{T} \mathbf{P}^{-1} \mathbf{x}(t),
$$


where $\mathbf{P} \in R^{n_{\mathbf{x}} \cdot n_{\mathbf{x}}}$ is a positive-definite matrix which needs to be designed later.

Define a transformation matrix $(\mathbf{M})$ and the associated vector (v) as

$$
\begin{aligned}
& \mathbf{M}=\left[\begin{array}{c}
\mathbf{M}_{1} \\
\mathbf{M}_{2}
\end{array}\right]=\left[\begin{array}{c}
\left(\mathbf{B}_{\perp}^{T} \mathbf{P} \mathbf{B}_{\perp}\right)^{-1} \mathbf{B}_{\perp}^{T} \\
\left(\mathbf{B}_{2}^{\mathrm{T}} \mathbf{P}^{-1} \mathbf{B}_{2}\right)^{-1} \mathbf{B}_{2}^{T} \mathbf{P}^{-1}
\end{array}\right], \\
& \mathbf{v}=\left[\begin{array}{c}
\mathbf{v}_{1} \\
\mathbf{v}_{2}
\end{array}\right]=\mathbf{M x},
\end{aligned}
$$

where $\mathbf{v}_{1} \in R^{n_{\mathbf{x}}-n_{\mathrm{u}}}$ and $\mathbf{v}_{2} \in R^{n_{\mathrm{u}}}$.

The "regular form" of the original system (15) is then represented as follows

$$
\begin{aligned}
{\left[\begin{array}{c}
\dot{\mathbf{v}}_{1}(t) \\
\dot{\mathbf{v}}_{2}(t)
\end{array}\right]=} & \left(\begin{array}{cc}
\mathbf{M}_{1}[\mathbf{A}(\Theta)+\Delta \mathbf{A}(\Theta)] \mathbf{P} \mathbf{B}_{\perp} & \mathbf{M}_{1}[\mathbf{A}(\Theta)+\Delta \mathbf{A}(\Theta)] \mathbf{B}_{2} \\
\mathbf{M}_{2}[\mathbf{A}(\Theta)+\Delta \mathbf{A}(\Theta)] \mathbf{P} \mathbf{B}_{\perp} & \mathbf{M}_{2}[\mathbf{A}(\Theta)+\Delta \mathbf{A}(\Theta)] \mathbf{B}_{2}
\end{array}\right) \\
& \cdot\left[\begin{array}{c}
\mathbf{v}_{1}(t) \\
\mathbf{v}_{2}(t)
\end{array}\right]+\left[\begin{array}{c}
\mathbf{M}_{1} \mathbf{B}_{1}(\Theta) \\
\mathbf{M}_{2} \mathbf{B}_{1}(\Theta)
\end{array}\right] \mathbf{w}(t)+\left[\begin{array}{c}
0 \\
\mathbf{M}_{2} \mathbf{B}_{2}
\end{array}\right] \mathbf{u}(t) .
\end{aligned}
$$

Corresponding to the regular form, the reduced-order sliding mode dynamics on the sliding surface $s(t)=0$ with dimension of $n_{\mathbf{x}}-n_{\mathbf{u}}$ can be obtained as $[22,23]$

$$
\begin{aligned}
& \dot{\mathbf{v}}_{1}(t)=\underbrace{\mathbf{M}_{1}[\mathbf{A}(\Theta)+\Delta \mathbf{A}(\Theta)] \mathbf{P B} \mathbf{B}_{\perp}}_{A_{c}} \mathbf{v}_{1}(t)+\underbrace{\mathbf{M}_{1} \mathbf{B}_{1}(\Theta)}_{B_{c}} \mathbf{w}(t), \\
& \mathbf{z}(t)=\underbrace{\mathbf{C}_{1}(\Theta) \mathbf{P B}_{\perp}}_{C_{c}} \mathbf{v}_{1}(t)+\mathbf{D}_{1}(\Theta) \mathbf{w}(t) .
\end{aligned}
$$

In the following, a robust stability criterion will be proposed to guarantee the existence of the sliding surface (18) under the existing of $\Delta \mathbf{A}(\Theta)$ and $\mathbf{w}(t)$.

Theorem 1. Given a scalar $\gamma>0$, the switching surface $s(t)$ exists and the sliding mode dynamics in (21) is admissible with bounded $L_{2}$ gain performance $\gamma$, that is, the system in the sliding mode is robust stable and has an $L_{2}$ gain less than $\gamma$ for all admissible uncertainties $\Delta \mathbf{A}(\Theta)$ and for all exogenous input $\mathbf{w}(t) \in L_{2}[0, \infty)$, if there exist matrices $\mathbf{P}>0, \mathbf{G}_{11}, \mathbf{G}_{21}, \ldots$, $\mathbf{G}_{51}, \mathbf{G}_{61}, \mathbf{G}_{12}, \mathbf{G}_{22}, \ldots, \mathbf{G}_{52}, \mathbf{G}_{62}$, and a scalar $\sigma>0$ such that for all $\Theta$

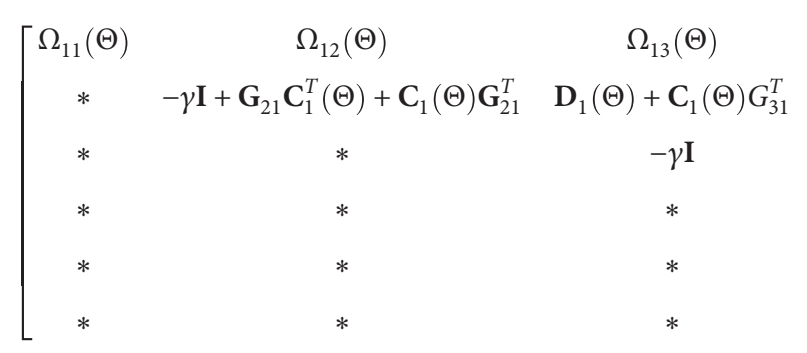

where

$$
\begin{aligned}
& \left.\Omega_{11}(\Theta)=\mathbf{G}_{11} \mathbf{A}(\Theta)\right)^{T} \mathbf{B}_{\perp}+\mathbf{B}_{\perp}^{T} \mathbf{A}(\Theta) \mathbf{G}_{11}^{T}+\varepsilon_{\Delta}^{2} \mathbf{G}_{12} \mathbf{B}_{\perp}+\varepsilon_{\Delta}^{2} \mathbf{B}_{\perp}^{T} \mathbf{G}_{12}^{T}, \\
& \Omega_{12}(\Theta)=\mathbf{B}_{\perp}^{T} \mathbf{A}(\Theta) \mathbf{G}_{21}^{T}+\varepsilon_{\Delta}^{2} \mathbf{B}_{\perp}^{T} \mathbf{G}_{22}^{T}+\mathbf{G}_{11} \mathbf{C}_{1}(\Theta)^{T} \\
& \Omega_{13}(\Theta)=\mathbf{B}_{\perp}^{T} \mathbf{B}_{1}(\Theta)+\mathbf{B}_{\perp}^{T} \mathbf{A}(\Theta) \mathbf{G}_{31}^{T}+\varepsilon_{\Delta}^{2} \mathbf{B}_{\perp}^{T} \mathbf{G}_{32}^{T} \\
& \Omega_{15}(\Theta)=\mathbf{B}_{\perp}^{T} \mathbf{P}+\varepsilon_{\Delta}^{2} \mathbf{B}_{\perp}^{T} \mathbf{G}_{52}^{T}+\mathbf{B}_{\perp}^{T} \mathbf{A}(\Theta) \mathbf{G}_{51}^{T}-\mathbf{G}_{11} \\
& \Omega_{14}(\Theta)=\mathbf{B}_{\perp}^{T} \mathbf{A}(\Theta) \mathbf{G}_{41}^{T}+\varepsilon_{\Delta}^{2} \mathbf{B}_{\perp}^{T} \mathbf{G}_{42}^{T}+\mathbf{G}_{11} \\
& \Omega_{16}(\Theta)=\frac{1}{2} \sigma \mathbf{B}_{\perp}^{T}+\mathbf{B}_{\perp}^{T} \mathbf{A}(\Theta) \mathbf{G}_{61}^{T}+\varepsilon_{\Delta}^{2} \mathbf{B}_{\perp}^{T} \mathbf{G}_{62}^{T}-\mathbf{G}_{12} .
\end{aligned}
$$

$$
\left.\begin{array}{ccc}
\Omega_{14}(\Theta) & \Omega_{15}(\Theta) & \Omega_{16}(\Theta) \\
\mathbf{G}_{21}+\mathbf{C}_{1}(\Theta) \mathbf{G}_{41}^{T} & -\mathbf{G}_{21}+\mathbf{C}_{1}(\Theta) G_{51}^{T} & -\mathbf{G}_{22}+\mathbf{C}_{1}(\Theta) G_{61}^{T} \\
\mathbf{G}_{31} & -\mathbf{G}_{31} & -\mathbf{G}_{32} \\
-\sigma \mathbf{I}+\mathbf{G}_{41}+\mathbf{G}_{41}^{T} & -\mathbf{G}_{41}+\mathbf{G}_{51}^{T} & -\mathbf{G}_{42}+\mathbf{G}_{61}^{T} \\
* & -\mathbf{G}_{51}-\mathbf{G}_{51}^{T} & -\mathbf{G}_{52}-\mathbf{G}_{61}^{T} \\
* & * & -\mathbf{G}_{62}-\mathbf{G}_{62}^{T}
\end{array}\right]<0,
$$

Proof. By using the bounded real lemma [12], the system (21) satisfies the robust $L_{2}$ performance requirement if and only if there exists a positive-define matrix $X_{c}$, such that

$$
\left[\begin{array}{ccc}
A_{c}^{T} X_{c}+X_{c} A_{c} & X_{c} B_{c} & C_{c}^{T} \\
* & -\gamma I & \mathbf{D}_{1}(\Theta)^{T} \\
* & * & -\gamma I
\end{array}\right]<0
$$

By selecting $X_{c}=\mathbf{B}_{\perp}^{T} \mathbf{P} \mathbf{B}_{\perp}$, substituting (21) into inequality (24), and applying Lemmas 1 and 2, inequality (24) holds if

$$
\left[\begin{array}{ccc}
\mathbf{B}_{\perp}^{T}\left[\mathbf{A}(\Theta) \mathbf{P}+\mathbf{P A}(\Theta)^{T}\right] \mathbf{B}_{\perp}+\sigma \varepsilon_{\triangle}^{2} \mathbf{B}_{\perp}^{T} \mathbf{B}_{\perp}+\frac{1}{\sigma} \mathbf{B}_{\perp}^{T} \mathbf{P} \mathbf{P} \mathbf{B}_{\perp} & \mathbf{B}_{\perp}^{T} \mathbf{B}_{1}(\Theta) & \mathbf{B}_{\perp}^{T} \mathbf{P} \mathbf{C}_{1}(\Theta)^{T} \\
* & -\gamma \mathbf{I} & \mathbf{D}_{1}(\Theta)^{T} \\
* & * & -\gamma \mathbf{I}
\end{array}\right]<0 .
$$


According to Schur complement formula and elementary transformation, inequality (25) is equivalent to

$$
\left[\begin{array}{cccc}
\mathbf{B}_{\perp}^{T}\left[\mathbf{A}(\Theta) \mathbf{P}+\mathbf{P A}(\Theta)^{T}\right] \mathbf{B}_{\perp}+\sigma \varepsilon_{\Delta}^{2} \mathbf{B}_{\perp}^{T} \mathbf{B}_{\perp} & \mathbf{B}_{\perp}^{T} \mathbf{P C}_{1}(\Theta)^{T} & \mathbf{B}_{\perp}^{T} \mathbf{B}_{1}(\Theta) & \mathbf{B}_{\perp}^{T} \mathbf{P} \\
* & -\gamma \mathbf{I} & \mathbf{D}_{1}(\Theta) & 0 \\
* & * & -\gamma \mathbf{I} & 0 \\
* & * & * & -\sigma \mathbf{I}
\end{array}\right]<0 .
$$

Inequality (26) can be further expressed as

$$
\left[\begin{array}{c}
\mathbf{I} \\
\mathbf{N}
\end{array}\right]^{T}\left[\begin{array}{cccccc}
0 & 0 & \mathbf{B}_{\perp}^{T} \mathbf{B}_{1}(\Theta) & 0 & \mathbf{B}_{\perp}^{T} \mathbf{P} & \frac{1}{2} \sigma \mathbf{B}_{\perp}^{T} \\
* & -\gamma \mathbf{I} & \mathbf{D}_{1}(\Theta) & 0 & 0 & 0 \\
* & * & -\gamma \mathbf{I} & 0 & 0 & 0 \\
* & * & * & -\sigma \mathbf{I} & 0 & 0 \\
* & * & * & * & 0 & 0 \\
* & * & * & * & * & 0
\end{array}\right]\left[\begin{array}{c}
\mathbf{I} \\
\mathbf{N}
\end{array}\right]<0,
$$

where

$$
\mathbf{N}=\left[\begin{array}{cccc}
\mathbf{A}(\Theta)^{T} \mathbf{B}_{\perp} & \mathbf{C}_{1}(\Theta)^{T} & 0 & \mathbf{I} \\
\varepsilon_{\Delta}^{2} \mathbf{B}_{\perp} & 0 & 0 & 0
\end{array}\right]
$$

By using Lemma 3, we have

$$
\left[\begin{array}{cccccc}
0 & 0 & \mathbf{B}_{\perp}^{T} \mathbf{B}_{1}(\Theta) & 0 & \mathbf{B}_{\perp}^{T} \mathbf{P} & \frac{1}{2} \sigma \mathbf{B}_{\perp}^{T} \\
* & -\gamma \mathbf{I} & \mathbf{D}_{1}(\Theta) & 0 & 0 & 0 \\
* & * & -\gamma \mathbf{I} & 0 & 0 & 0 \\
* & * & * & -\sigma \mathbf{I} & 0 & 0 \\
* & * & * & * & 0 & 0 \\
* & * & * & * & * & 0
\end{array}\right]
$$

where

$$
\mathbf{G}=\left[\begin{array}{llllll}
\mathbf{G}_{11}^{T} & \mathbf{G}_{21}^{T} & \mathbf{G}_{31}^{T} & \mathbf{G}_{41}^{T} & \mathbf{G}_{51}^{T} & \mathbf{G}_{61}^{T} \\
\mathbf{G}_{12}^{T} & \mathbf{G}_{22}^{T} & \mathbf{G}_{32}^{T} & \mathbf{G}_{42}^{T} & \mathbf{G}_{52}^{T} & \mathbf{G}_{62}^{T}
\end{array}\right]^{T}
$$

After matrix decomposition and combination, we can obtain the final inequality (22). This completes the proof.
Remark 5. In inequality (22), G is considered as a fullblock matrix to get the feasible solution of LMIs. We may also simplify $\mathbf{G}$ by setting $\mathbf{G}_{i j}=\mathbf{0}$ or $\mathbf{G}_{i j}=\mathbf{I}$ partly to reduce computational complexity. But this will bring conservatism definitely.

We notice that the inequality (22) has the infinite number of LMI constraints. Therefore, the main additional challenge in the LPV system sliding surface existence is hence the reduction of these to finite-dimensional LMIs that can be solved via semidefinite programming (SDP). Here, a possible approach is to transform the nominal LPV system (15) into a convex polytopic one by TP model transformation. Then based on the properties of a convex set, it can be concluded that these infinite constraints will hold for all parameter trajectories if and only if it holds for the finite vertex systems.

In TP model transformation, the step of making highorder singular value decomposition (HOSVD) to a tensor is regarded as the crucial point [35]. When constructing a polytopic LPV model by HOSVD, its computational complexity is directly proportional to the number of the reserved singular values. In the work of [36], we can find the details of HOSVD about computational complexity relaxation and the trade-off between approximation accuracy and complexity.

Now, we consider the following system matrix

$$
\mathbf{S}(\Theta)=\left[\begin{array}{ll}
\mathbf{A}(\Theta) & \mathbf{B}_{1}(\Theta) \\
\mathbf{C}_{1}(\Theta) & \mathbf{D}_{1}(\Theta)
\end{array}\right] .
$$

Using TP model transformation and discarding the smaller singular values as well as their corresponding singular vectors, one obtains a higher order form as

$$
\mathbf{S}(\Theta) \approx \sum_{i_{1}=1}^{I_{1}} \sum_{i_{1}=1}^{I_{2}} \ldots \sum_{i_{N}}^{I_{N}} \prod_{n=1}^{n_{\theta}} \mathrm{w}_{n, i_{n}}\left(\theta_{n}\right) \mathbf{S}_{i_{1}, i_{2}, \ldots, i_{N}},
$$

with $\sum_{i_{n}=1}^{I_{n}} \mathrm{w}_{n, i_{n}}\left(\theta_{n}\right)=1, \forall n, \theta_{n}$, and $\mathrm{w}_{n, i_{n}}\left(\theta_{n}\right) \geq 0, \forall n, \theta_{n}, i_{n}$. In (32), $I_{1}, I_{2}, \ldots, I_{N}$ stand for the number of the reserved singular values along each dimension.

The tensor $\mathbf{S}(\Theta)$ is then approximately constructed from finite LTI vertex systems $\mathbf{S}_{i_{1}, i_{2}, \ldots, i_{N}}$ and the weight function 
$\mathrm{w}_{n, i_{n}}\left(\theta_{n}\right)$ which is obtained through reserved singular vectors. Here, to guarantee that the resulting LTI vertex systems form a convex hull of the LPV system, we use the canonical form to transform the weight functions.
Consequently, we only need to consider finite number of LMI constraints corresponding to the obtained vertex system $\mathbf{S}_{i_{1}, i_{2}, \ldots, i_{N}}$ for the controller design, which means that inequality (22) in Theorem 1 can be rewritten as

$$
\left[\begin{array}{ccc}
\boldsymbol{\Omega}_{11 i} & \Omega_{12 i} & \Omega_{13 i} \\
* & -\gamma \mathbf{I}+\mathbf{G}_{21} \mathbf{C}_{1 i}^{T}+\mathbf{C}_{1 i} \mathbf{G}_{21}^{T} & \mathbf{D}_{1 i}+\mathbf{C}_{1 i} G_{31}^{T} \\
* & * & -\gamma \mathbf{I} \\
* & * & * \\
* & * & * \\
* & * & *
\end{array}\right.
$$

where

$$
\begin{aligned}
& \Omega_{11 i}=\mathbf{G}_{11} \mathbf{A}_{i}^{T} \mathbf{B}_{\perp}+\mathbf{B}_{\perp}^{T} \mathbf{A}_{i} \mathbf{G}_{11}^{T}+\varepsilon_{\Delta}^{2} \mathbf{G}_{12} \mathbf{B}_{\perp}+\varepsilon_{\Delta}^{2} \mathbf{B}_{\perp}^{T} \mathbf{G}_{12}^{T}, \\
& \Omega_{12 i}=\mathbf{B}_{\perp}^{T} \mathbf{A}_{i} \mathbf{G}_{21}^{T}+\varepsilon_{\Delta}^{2} \mathbf{B}_{\perp}^{T} \mathbf{G}_{22}^{T}+\mathbf{G}_{11} \mathbf{C}_{1 i}^{T}, \\
& \Omega_{13 i}=\mathbf{B}_{\perp}^{T} \mathbf{B}_{1 i}+\mathbf{B}_{\perp}^{T} \mathbf{A}_{i} \mathbf{G}_{31}{ }^{T}+\varepsilon_{\Delta}^{2} \mathbf{B}_{\perp}^{T} \mathbf{G}_{32}^{T}, \\
& \Omega_{14 i}=\mathbf{B}_{\perp}^{T} \mathbf{A}_{i} \mathbf{G}_{41}{ }^{T}+\varepsilon_{\Delta}^{2} \mathbf{B}_{\perp}^{T} \mathbf{G}_{42}^{T}+\mathbf{G}_{11}, \\
& \Omega_{15 i}=\mathbf{B}_{\perp}^{T} \mathbf{P}_{i}+\varepsilon_{\Delta}^{2} \mathbf{B}_{\perp}^{T} \mathbf{G}_{52}^{T}+\mathbf{B}_{\perp}^{T} \mathbf{A}_{i} \mathbf{G}_{51}{ }^{T}-\mathbf{G}_{11}, \\
& \Omega_{16 i}=\frac{1}{2} \sigma \mathbf{B}_{\perp}^{T}+\mathbf{B}_{\perp}^{T} \mathbf{A}_{i} \mathbf{G}_{61}{ }^{T}+\varepsilon_{\Delta}^{2} \mathbf{B}_{\perp}^{T} \mathbf{G}_{62}^{T}-\mathbf{G}_{12} .
\end{aligned}
$$

Remark 6. We apply Lemma 3 to eliminate the coupling between the Lyapunov function matrix and the system matrix. Then for the purpose of reducing conservatism, different Lyapunov functions at each of the vertices of the polytope system should be employed. The details and verifications can be found in $[35,37]$. Furthermore, if the solution $\mathbf{P}_{i_{1}, i_{2}, \ldots, i_{N}}$ exists at each of the vertices, then the parameter-dependent Lyapunov function can be obtained as

$$
\mathbf{P}(\Theta)=\sum_{i_{1}=1}^{I_{1}} \sum_{i_{1}=1}^{I_{2}} \ldots \sum_{i_{N}}^{I_{N}} \prod_{n=1}^{N} \mathrm{w}_{n, i_{n}}\left(\theta_{n}\right) \mathbf{P}_{i_{1}, i_{2}, \ldots, i_{N}} .
$$

4.2. SMC Law Synthesis. In this section, we shall synthesize an FSMC law, by which the trajectories of the LPV system in (15) can be driven onto the prespecified switching surface $s$ $(t)=0$ in finite time and then are maintained there for all subsequent time.

Theorem 2. Suppose that the switching surface function (18) exists, namely, the LMIs in (33) is feasible for $\mathbf{P}_{i_{1}, i_{2}, \ldots, i_{N}}$. Then the trajectories of system (15) can be driven onto the switching surface $s(t)=0$ by the following sliding mode control law:

$$
\left.\begin{array}{ccc}
\Omega_{14 i} & \Omega_{15 i} & \Omega_{16 i} \\
\mathbf{G}_{21}+\mathbf{C}_{1 i} \mathbf{G}_{41}^{T} & -\mathbf{G}_{21}+\mathbf{C}_{1 i} G_{51}^{T} & -\mathbf{G}_{22}+\mathbf{C}_{1 i} \mathbf{G}_{61}^{T} \\
\mathbf{G}_{31} & -\mathbf{G}_{31} & -\mathbf{G}_{32} \\
-\sigma \mathbf{I}+\mathbf{G}_{41}+\mathbf{G}_{41}^{T} & -\mathbf{G}_{41}+\mathbf{G}_{51}^{T} & -\mathbf{G}_{42}+\mathbf{G}_{61}^{T} \\
* & -\mathbf{G}_{51}-\mathbf{G}_{51}^{T} & -\mathbf{G}_{52}-\mathbf{G}_{61}^{T} \\
* & * & -\mathbf{G}_{62}-\mathbf{G}_{62}^{T}
\end{array}\right]<0,
$$

$$
\begin{aligned}
\mathbf{u}(t)= & -\mathbf{M}_{2}(\Theta)\left[\mathbf{A}(\Theta)+\mathbf{P}^{\prime}(\Theta) \mathbf{P}(\Theta)^{-1}\left[\mathbf{B}_{2} \mathbf{M}_{2}(\Theta)-\mathbf{I}\right]\right] \mathbf{x}(t) \\
& -\eta(\Theta) \operatorname{sign}\left(\mathbf{M}_{2}(\Theta) \mathbf{x}(t)\right),
\end{aligned}
$$

where $\mathbf{M}_{2}(\Theta)$ is described in (19) with $\mathbf{P}^{-1}$ turning into $\mathbf{P}(\Theta)^{-1}$. $\mathbf{P}^{\prime}(\Theta)$ has the form as $\mathbf{P}^{\prime}(\Theta)=\sum_{i_{1}=1}^{I_{1}} \sum_{i_{2}=1}^{I_{2}} \cdots \sum_{i_{N}=1}^{I_{N}}$ $\left(\prod_{n=1}^{N} \mathrm{w}_{n, i_{n}}\left(\theta_{n}\right)\right)^{\prime} \mathbf{P}_{i_{1}, i_{2}, \ldots, i_{N}}$ and $\eta(\Theta)$ is designed as

$$
\eta(\Theta)=\mu+\varepsilon_{\Delta}\left\|\mathbf{M}_{2}(\Theta)\right\|\|\mathbf{x}\|+\varepsilon_{w}\left\|\mathbf{M}_{2}(\Theta) \mathbf{B}_{1}(\Theta)\right\|,
$$

where $\mu$ is a positive scalar.

Proof. As the surface is defined as $s(t)=0$, we choose the following parameter-dependent Lyapunov function

$$
\begin{aligned}
V(t) & =\frac{1}{2} s(t)^{T}\left(\left(\mathbf{B}_{2}^{\mathrm{T}} \mathbf{P}(\Theta)^{-1} \mathbf{B}_{2}\right)^{-1}\right)^{T}\left(\mathbf{B}_{2}^{\mathrm{T}} \mathbf{P}(\Theta)^{-1} \mathbf{B}_{2}\right)^{-1} s(t) \\
& =\frac{1}{2} \mathbf{x}^{T} \mathbf{M}_{2}(\Theta)^{T} \mathbf{M}_{2}(\Theta) \mathbf{x} .
\end{aligned}
$$

Taking the derivative of $V(t)$, considering the SMC law in (36) and noting $\mathbf{M}_{2}(\Theta) \mathbf{B}_{2}=\mathbf{I}$ and $\mathbf{M}_{2}^{\prime}(\Theta)=\mathbf{M}_{2}(\Theta) \mathbf{P}^{\prime}(\Theta)$ $\mathbf{P}(\Theta)^{-1}\left[\mathbf{B}_{2} \mathbf{M}_{2}(\Theta)-\mathbf{I}\right]$, we have

$$
\begin{gathered}
\dot{V}(t)=\mathbf{x}^{T} \mathbf{M}_{2}(\Theta)^{T}\left[\mathbf{M}_{2}(\Theta) \Delta \mathbf{A}(\Theta) \mathbf{x}+\mathbf{M}_{2}(\Theta) \mathbf{B}_{1}(\Theta) \mathbf{w}\right. \\
\left.-\eta(\Theta) \operatorname{sign}\left(\mathbf{M}_{2}(\Theta) \mathbf{x}\right)\right] .
\end{gathered}
$$

In addition, we notice that $\mathbf{x}^{T} \mathbf{M}_{2}(\Theta)^{T} \operatorname{sign}\left(\mathbf{M}_{2}(\Theta) \mathbf{x}\right) \geq$ $\left\|\mathbf{M}_{2}(\Theta) \mathbf{x}\right\|$ and $\eta(\Theta)>0$. Thus,

$$
\begin{aligned}
\dot{V}(t) \leq & \left\|\mathbf{x}^{T} \mathbf{M}_{2}(\Theta)^{T}\right\| \\
\cdot & \cdot\left[\varepsilon_{\Delta}\left\|\mathbf{M}_{2}(\Theta)\right\|\|\mathbf{x}\|+\varepsilon_{w}\left\|\mathbf{M}_{2}(\Theta) \mathbf{B}_{1}(\Theta)\right\|-\eta(\Theta)\right] .
\end{aligned}
$$



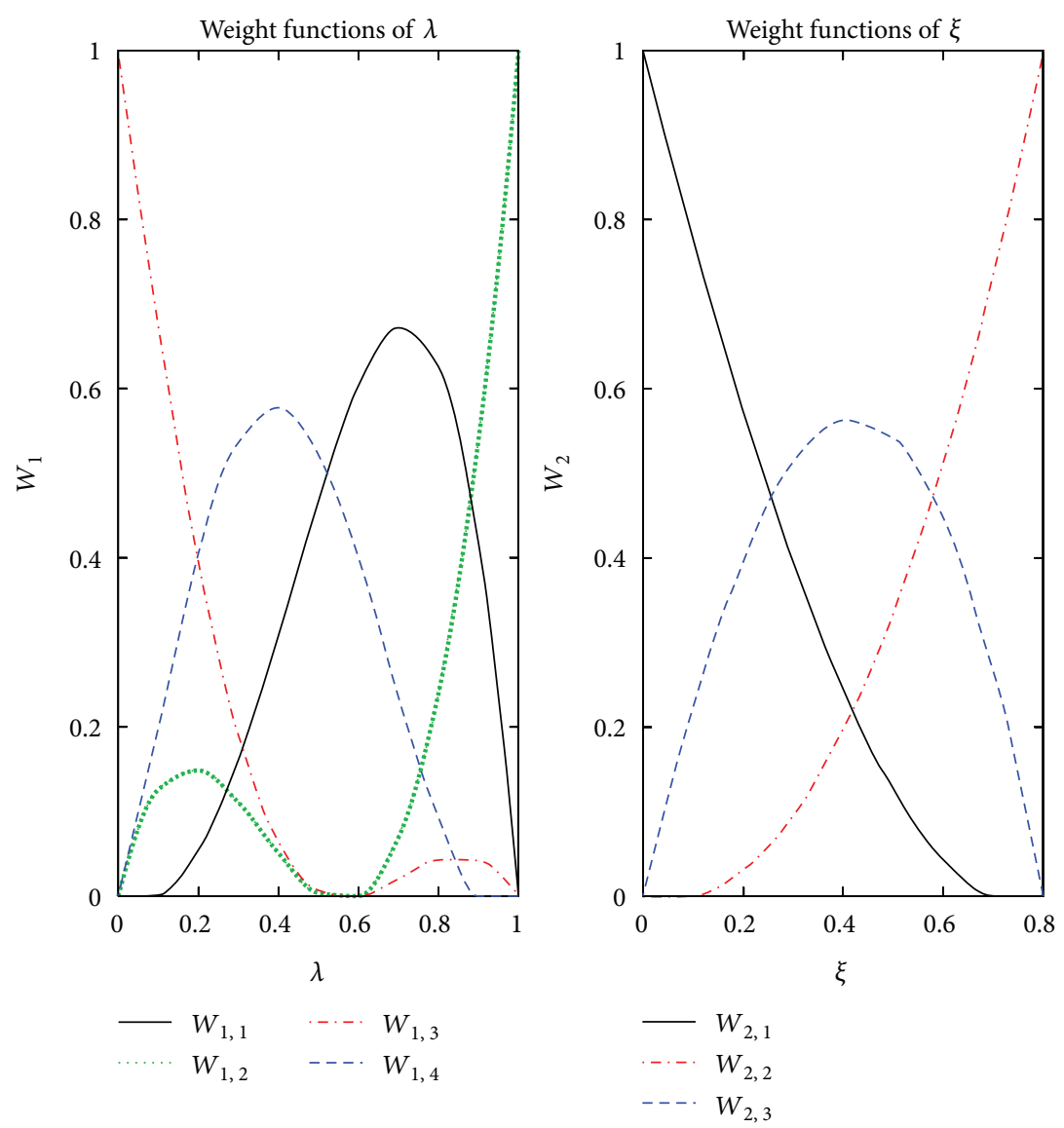

Figure 10: Weight functions of the convex model.

Substituting (37), inequality (40) can be further reduced to

$$
\dot{V}(t) \leq-\mu\left\|\mathbf{x}^{T} \mathbf{M}_{2}(\Theta)^{T}\right\|=-\mu V^{1 / 2}(t) .
$$

By using Lemma 4, it can be seen from (41) that there exists a time $t^{*} \in\left(0, V^{1 / 2}(0) / \mu\right]$ such that $V(t)=0$ and consequently $s(t)=0$ when $t \geq t^{*}$. This means that the system trajectories can be driven onto the predefined switching surface in a finite time, thereby completing the proof.

Remark 7. To deal with the problems introduced by chattering phenomenon, we replace the switching term $\operatorname{sign}(\bullet)$ with $\bullet / \| \bullet+\beta$, where $\beta$ is an adjustable scalar. Then, one can tune the gain $\mu$ and $\beta$ to get a better robust performance and reduce the chattering simultaneously.

\section{Simulation}

Having formulated the approach to modeling of morphing dynamic equations and FSMC-LPV controller synthesis, we are in a position to verify the effectiveness and performance of the presented algorithm when applied in the longitudinal nonlinear model. To this end, this section is intended to illustrate that the controller can make the altitude and speed remain constant during morphing process when subjected to parameter uncertainty and external disturbance.

To obtain a standard realization of the LPV plant from system (14) to system (15), we consider an $L_{2}$ gain performance minimization problem with a performance output $\mathbf{z}(t)=\left[I_{V} I_{h}\right]^{T}$, where $I_{V}=\int e_{V} d t$ and $I_{h}=\int e_{h} d t$ represent the error integrals of speed and altitude, respectively. Meantime, for turning $\mathbf{B}_{2}$ into a constant matrix, two additional state variables are introduced to filter inputs $\mathbf{u}=\left[\Delta \delta_{e}, \Delta \delta_{t}\right]^{T}$. Here, we select the matrices of prefilter (16) as

$$
\begin{aligned}
& \mathbf{A}_{\mathbf{u}}=\left[\begin{array}{cc}
-0.5 & 1 \\
0 & -0.5
\end{array}\right], \\
& \mathbf{B}_{\mathbf{u}}=\left[\begin{array}{ll}
1 & 1
\end{array}\right]^{T}, \\
& \mathbf{C}_{\mathbf{u}}=\mathbf{I}_{2} .
\end{aligned}
$$

The rest of coefficient matrixes in (15) are set as $\mathbf{B}_{1}(\Theta)=$ $\left[\begin{array}{ll}\mathbf{I}_{3 \times 3} & 0\end{array}\right]^{T}, \mathbf{C}_{1}(\Theta)=\left[\begin{array}{ll}0 & \mathbf{I}_{2 \times 2}\end{array}\right]^{T}$, and $\mathbf{D}_{1}(\Theta)=0$.

In what follows, we calculate the parameter-dependent Lyapunov function $\mathbf{P}(\Theta)$ in Theorem 1. Considering the computational load and approximation accuracy, four singular values of $\lambda$ dimension and three singular values of $\xi$ dimension as well as their corresponding singular vectors are remained to obtain the minimal vertex systems. Therefore, it is concluded that the polynomial parameter- 

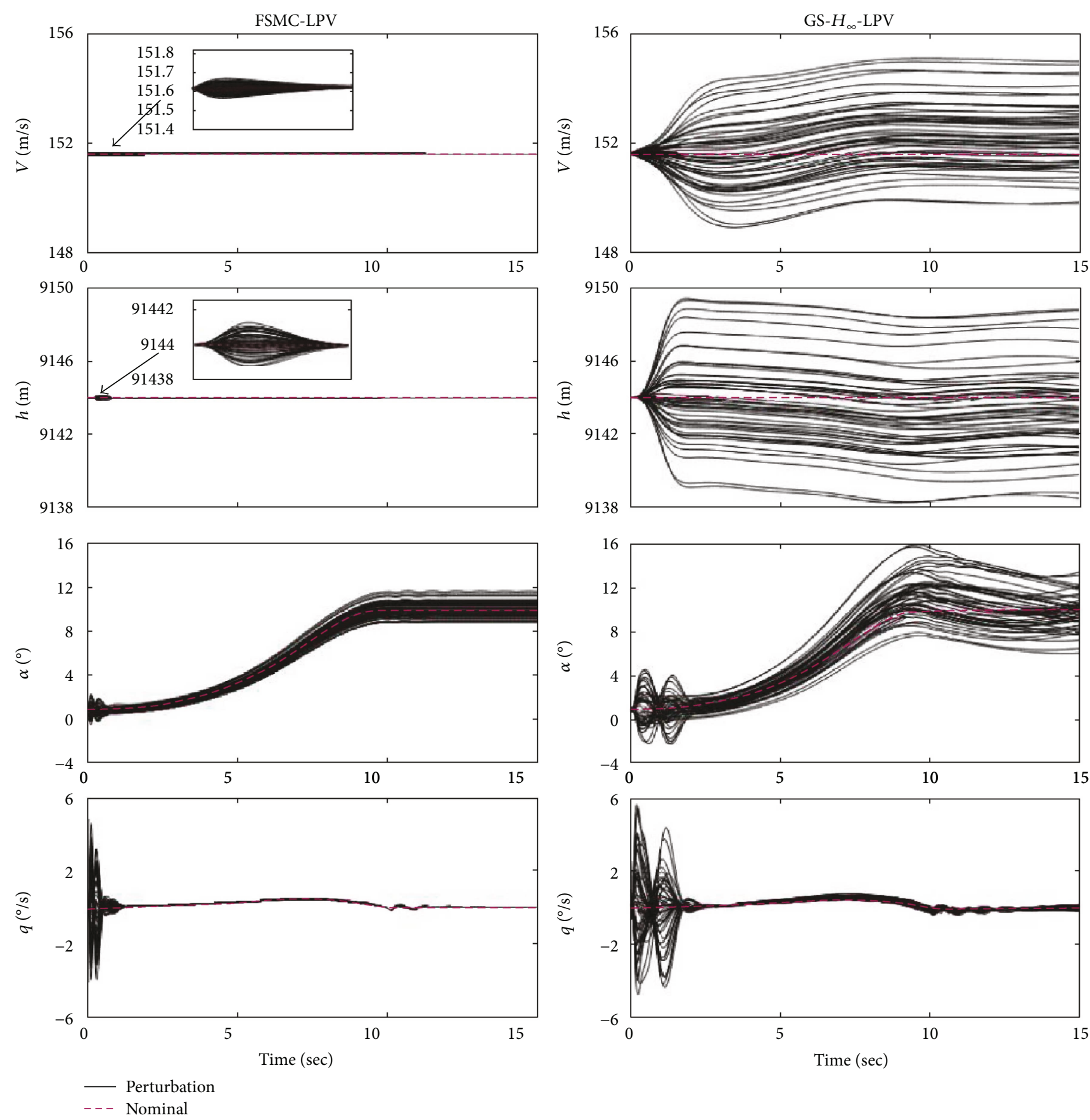

FIGURE 11: Closed-loop response during morphing process.

dependent LPV system (11) can be approximately presented in the convex polytopic form with minimum $4 \times 3=12 \mathrm{LTI}$ systems, that is,

$$
\begin{aligned}
\mathbf{S}(\lambda, \xi) & =\left[\begin{array}{ll}
\mathbf{A}(\lambda, \xi) & \mathbf{B}_{2}(\lambda, \xi)
\end{array}\right] \\
& \approx \sum_{i}^{4} \sum_{j}^{3} w_{1, i}(\lambda) w_{2, j}(\xi)\left[\begin{array}{ll}
\mathbf{A}_{i, j} & \mathbf{B}_{2_{i, j}}
\end{array}\right]
\end{aligned}
$$

where the normalized weighting functions are given in Figure 10.
By using the YALMIP interface with the SDP solver SeduMi toolbox in MATLAB, we can calculate the minimal performance $\gamma_{\min }=4.6567$ in Theorem 1 . However, optimality is not desirable in this case since it is achieved at the expense of large entry values in the concerned matrixes. To prevent this phenomenon, we use a slightly higher value of $\gamma$. In this simulation, the desired performance is chosen as $\gamma_{\text {subopt }}=5.0$. Then, the Lyapunov matrixes $\mathbf{P}_{i, j}$ can be calculated at each LTI vertex systems $\mathbf{S}(\lambda, \xi)_{i, j}$.

For the aircraft during both morphing state and fixed state, the concerned model uncertain parameters and 

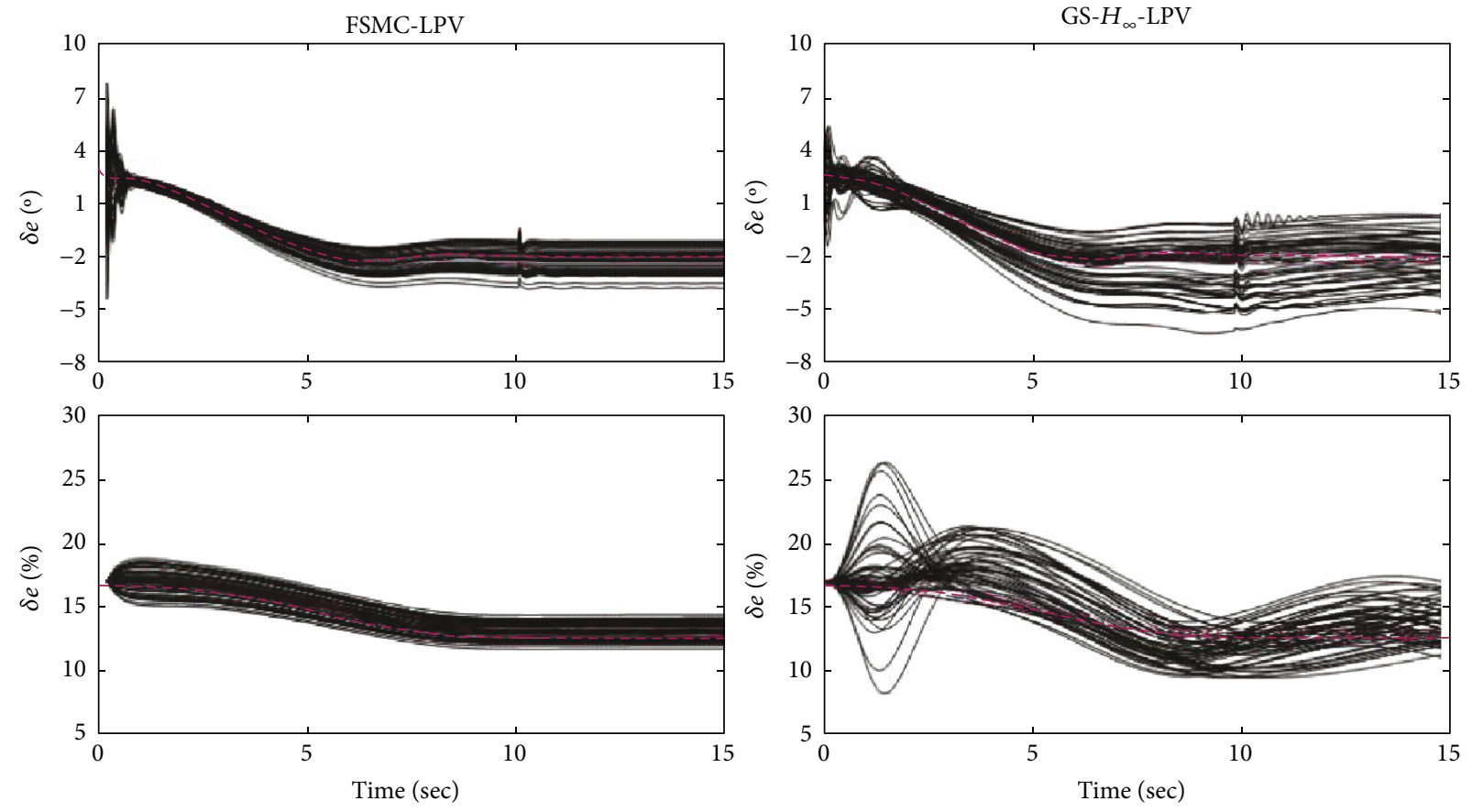

FIGURE 12: Closed-loop controller outputs.

external disturbance are given in (44). Based on these uncertainties, the controller parameters are designed in (45).

$$
\begin{aligned}
\Delta C_{L} & =\Delta C_{D}=\Delta C_{M}= \pm 30 \%, \\
\Delta m_{t} & =\Delta J_{y}= \pm 5 \%, \\
\Delta\left(\frac{1}{2} \rho V_{0}^{2}\right) & = \pm 20 \%, \\
d_{F_{V}} & =0.01 \sin (2 \pi t), \\
d_{F_{\alpha}} & =0.02 \sin (2 \pi t), \\
d_{M_{q}} & =0.05 \sin (2 \pi t), \\
\mu & =5.6, \\
\varepsilon^{w} & =0.2445, \\
\varepsilon_{\Delta} & =0.2, \\
\beta & =0.1 .
\end{aligned}
$$

Here, we consider the following case: morphing movement starts from configuration I to configuration II (see Figure 4) taking 10 seconds to complete. The energyoptimal motion trajectories of the sweep angle and span are just designed as Figure 7. The resulting FSMC with $L_{2}$ gain performance is applied at the morphing aircraft's wing transition phase. Comparative simulations are also developed to verify the robustness of the proposed controllers. Gain selfscheduled $H_{\infty}$ controllers (GS- $H_{\infty}$ ) can be designed for the same vertex LTI systems (43) based on the method which is derived from $[12,17]$, and, correspondingly, the suboptimal performance is chosen as $\gamma_{\text {subopt }}=6.0$.

In what follows, we perform 50 separate simulation experiments with different perturbation parameters for the
Table 2: Partial velocity of each body.

\begin{tabular}{cccccc}
\hline & $i=1$ & $i=2$ & $i=3$ & $i=4$ & $i=5$ \\
\hline $\mathbf{U}_{O_{b}}^{i(\mathbf{u})}$ & $\mathbf{e}_{\mathbf{x}}^{\mathbf{b}}$ & $\mathbf{e}_{\mathbf{x}}^{\mathbf{b}}$ & $\mathbf{e}_{\mathbf{x}}^{\mathbf{b}}$ & $\mathbf{e}_{\mathbf{x}}^{\mathbf{b}}$ & $\mathbf{e}_{\mathbf{x}}^{\mathbf{b}}$ \\
$\mathbf{U}_{O_{b}}^{i(\mathbf{w})}$ & $\mathbf{e}_{\mathbf{z}}^{\mathbf{b}}$ & $\mathbf{e}_{\mathbf{z}}^{\mathbf{b}}$ & $\mathbf{e}_{\mathbf{z}}^{\mathbf{b}}$ & $\mathbf{e}_{\mathbf{z}}^{\mathbf{b}}$ & $\mathbf{e}_{\mathbf{z}}^{\mathbf{b}}$ \\
$\mathbf{U}_{O_{b}}^{i(\mathbf{q})}$ & $-b \mathbf{e}_{\mathbf{z}}^{\mathbf{b}}$ & & $l_{\Delta} \sin \Lambda \mathbf{e}_{\mathbf{z}}^{\mathbf{b}}$ & $\left(l_{1} / 2\right) \sin \Lambda \mathbf{e}_{\mathbf{z}}^{\mathbf{b}}$ & $l_{\Delta} \sin \Lambda \mathbf{e}_{\mathbf{z}}^{\mathbf{b}}$ \\
$\mathbf{W}_{O_{b}}^{i(\mathbf{u})}$ & 0 & 0 & 0 & 0 & 0 \\
$\mathbf{W}_{O_{b}}^{i(\mathbf{w})}$ & 0 & 0 & 0 & 0 & 0 \\
$\mathbf{W}_{O_{b}}^{i(\mathbf{q})}$ & $\mathbf{e}_{\mathbf{y}}^{\mathbf{b}}$ & $\mathbf{e}_{\mathbf{y}}^{\mathbf{b}}$ & $\mathbf{e}_{\mathbf{y}}^{\mathbf{b}}$ & $\mathbf{e}_{\mathbf{y}}^{\mathbf{b}}$ & $\mathbf{e}_{\mathbf{y}}^{\mathbf{b}}$ \\
\hline
\end{tabular}

two controllers. At each group of two comparative experiments; however, the values of perturbation parameters are identical. The response of the aircraft in a closed-loop simulation is shown in Figure 11. In addition, Figure 12 gives the changes in elevator deflection and throttle.

It is clear that under parameter perturbation and external disturbances, the FSMC-LPV controller can guarantee the variation of speed being less than $0.1 \mathrm{~m} / \mathrm{s}$ and the altitude increases or decreases $0.2 \mathrm{~m}$ to the maximum during the wing morphing process. They can both converge to the previous value in a short time. Due to the uncertainties, the elevator deflection and the rate of pitch may change drastically at the beginning, but they are both within acceptable ranges. Since the wing area is reducing, the angle of attack will increase smoothly to achieve a new balance. It is obvious that the FSMC-LPV controller based on the morphing aircraft's linear model can eliminate the adverse effect of uncertainties caused by wing morphing and guarantee constant speed and altitude flight during the wing transition process. 
On the other hand, provided that there exist no parameters perturbation, conventional GS- $H_{\infty}$ LPV controller can perform as well as the FSMC-LPV controller. However, $H_{\infty}$ controller, although being a robust strategy especially for external disturbance, cannot restrain parameter perturbation effectively. This will cause the speed and altitude to fail and to remain in previous levels. Although the closed-loop system is stable, the changes in elevator deflection and throttle significantly increase during the morphing process, which may generate failures in the actuators. Overall, Figures 11 and 12 elaborate the control performances between the two controllers and we can conclude that the FSMC-LPV controller is more robust under parameter perturbation.

\section{Conclusion}

In this paper, a systematic method of LPV-based sliding mode controller synthesis is explored for a morphing aircraft with a variable sweep angle and a variable span. At first, the longitudinal dynamic model of the morphing aircraft is derived by using Kane method. Then the LPV model with mismatched uncertainties of the morphing aircraft is developed and it can describe the morphing phase's complex behavior. Therefore, the following controller synthesis can be based on the obtained LPV model. Secondly, we extend the sliding mode control of existing works for LTI systems via an LPV methodology. Sufficient condition in the form of LMIs has been established so as to ensure that the dynamics in the sliding mode are robustly admissible with a bounded $L_{2}$ gain performance. In order to avoid solving an infinite number of LMI constraints during the controller synthesis, we give a less conservative LMI existence condition by applying slack variables decoupling method and convex polytopic transformation. Furthermore, according to the parameter-dependent Lyapunov function stability analysis, it is verified that the system trajectories can be driven onto the predefined switching surface in a finite time by the synthesized FSMC. Finally, the sliding mode approach has been verified to maintain nominal performance and robustness in the face of parameter perturbation and external disturbance by the numerical simulations. The proposed sliding mode control synthesis for uncertain LPV system can be extended to cover a wide range of general LPV systems.

\section{Nomenclature}

$\begin{array}{ll}\Lambda: & \text { Sweep angle (deg) } \\ \Delta: & \text { Expansion of wingspan }(\mathrm{m}) \\ u, w: & \text { Scalar components of speed in body axes } \\ & (\mathrm{m} / \mathrm{s}) \\ q: & \text { Pitch angular velocity }(\mathrm{rad} / \mathrm{s}) \\ J_{x}, J_{y}, J_{z}: & \text { Moments of inertia of the fuselage }\left(\mathrm{kg} / \mathrm{s}^{2}\right) \\ J_{2}, J_{3}, J_{4}, J_{5}: & \text { Moments of inertia of the body } i\left(\mathrm{~kg} / \mathrm{s}^{2}\right) \\ l_{1}, l_{2}: & \text { Fixed length of inner wing and outer wing } \\ l_{\Delta}: & \text { (m) } \\ \alpha: & \text { The distance from } \mathrm{O}_{b} \text { to } \mathrm{O}_{3} \text { or } \mathrm{O}_{5}(\mathrm{~m}) \\ \theta: & \text { Angle of attack }(\mathrm{rad}) \\ m_{t}: & \text { Pitch angle }(\mathrm{rad})\end{array}$

$\begin{array}{ll}D, L, T: & \text { Drag, lift, and thrust }(\mathrm{N}) \\ g: & \text { Acceleration due to gravity }\left(\mathrm{m} / \mathrm{s}^{2}\right) \\ C_{D_{\alpha}}, C_{L}, C_{M}: & \text { The coefficients of drag, lift, and moment } \\ \delta_{e}: & \text { Elevator angular deflection (rad) } \\ \delta_{T}: & \text { Throttle (\%) } \\ T_{\delta_{T}}: & \text { Throttle coefficient } \\ C_{L_{\alpha=0}}, C_{L_{\alpha}}, C_{L_{\delta e}}: & \text { Lift force coefficient with respect to } \alpha=0 \\ C_{M_{\alpha=0}}, C_{M_{\alpha}}, C_{M_{\delta e}}: & \text { Pitching moment coefficient with respect } \\ M: & \text { to } \alpha=0, \alpha, \delta_{e} \\ V: & \text { Pitching moment }(\mathrm{N} \bullet \mathrm{m}) \\ h: & \text { Velocity (m/s) } \\ \rho: & \text { Altitude }(\mathrm{m}) \\ S_{w}: & \text { Air density }\left(\mathrm{kg} / \mathrm{m}^{3}\right) \\ b: & \left.\text { Wing area (m }{ }^{2}\right) \\ \lambda, \xi: & \text { The distance from } O_{b} \text { to } O_{1}(\mathrm{~m}) \\ & \text { Scheduling parameters. }\end{array}$

\section{Appendix}

\section{A. The Specific Definitions of Variables in Step 2 and Step 3 of Figure 3}

The generalized active force $\mathbf{F}_{O_{b}}^{i}$ of body $i$ can be given by

$$
\begin{aligned}
\mathbf{F}_{O_{b}}^{1}= & \left(T-m_{b} g \sin \theta+L \sin \alpha-D \cos \alpha\right) \mathbf{e}_{\mathbf{x}}^{\mathbf{b}} \\
& +\left(m_{b} g \cos \theta-D \sin \alpha-L \cos \alpha\right) \mathbf{e}_{\mathbf{z}}^{\mathbf{b},} \\
\mathbf{F}_{O_{b}}^{2}= & -m_{1} g \sin \theta \mathbf{e}_{\mathbf{x}}^{\mathbf{b}}+m_{1} g \cos \theta \mathbf{e}_{\mathbf{z}}^{\mathbf{b}}, \\
\mathbf{F}_{O_{b}}^{3}= & -m_{2} g \sin \theta \mathbf{e}_{\mathbf{x}}^{\mathbf{b}}+m_{2} g \cos \theta \mathbf{e}_{\mathbf{z}}^{\mathbf{b}}, \\
\mathbf{F}_{O_{b}}^{4}= & -m_{1} g \sin \theta \mathbf{e}_{\mathbf{x}}^{\mathbf{b}}+m_{1} g \cos \theta \mathbf{e}_{\mathbf{z}}^{\mathbf{b}}, \\
\mathbf{F}_{O_{b}}^{5}= & -m_{2} g \sin \theta \mathbf{e}_{\mathbf{x}}^{\mathbf{b}}+m_{2} g \cos \theta \mathbf{e}_{\mathbf{z}}^{\mathbf{b}} .
\end{aligned}
$$

The generalized active moment $\mathbf{M}_{O_{b}}^{i}$ can be given by

$$
\begin{aligned}
& \mathbf{M}_{O_{b}}^{1}=M \mathbf{e}_{\mathbf{y}}^{\mathbf{b}}, \\
& \mathbf{M}_{O_{b}}^{2}=\mathbf{M}_{O_{b}}^{3}=\mathbf{M}_{O_{b}}^{4}=\mathbf{M}_{O_{b}}^{5}=0 .
\end{aligned}
$$

The partial velocities $\mathbf{U}_{O_{b}}^{i(\sim)}$ and $\mathbf{W}_{O_{b}}^{i(\sim)}$ of each body $i$ are defined in Table 2.

The generalized inertial force $\mathbf{F}_{O_{b}}^{i}$ and the generalized inertial moment $\mathbf{M}_{\mathrm{O}_{b}}^{i}$ of body $i$ can be obtained as

$$
\begin{aligned}
\tilde{\mathbf{F}}_{O_{b}}^{i} & =-m^{i} \mathbf{a}_{O_{b}}^{i}=-m^{i}\left(\frac{\delta \mathbf{v}_{O_{b}}^{i}}{\delta t}+\boldsymbol{\omega}_{O_{b}}^{i} \times \mathbf{v}_{O_{b}}^{i}\right), \\
\tilde{\mathbf{M}}_{O_{b}}^{i} & =-\mathbf{J}_{O_{b}}^{i} \frac{\delta \boldsymbol{\omega}_{O_{b}}^{i}}{\delta t}-\boldsymbol{\omega}_{O_{b}}^{i} \times\left(\mathbf{J}_{O_{b}}^{i} \boldsymbol{\omega}_{O_{b}}^{i}\right),
\end{aligned}
$$

where $m^{i}$ is the mass of body. $\mathbf{a}_{O_{b}}^{i}$ is the acceleration of the center of mass. $\mathbf{v}_{O_{b}}^{i}$ is the velocity. $\mathbf{J}_{O_{b}}^{i}$ is the inertia matrix. $\boldsymbol{\omega}_{\mathrm{O}_{b}}^{i}$ is the rotation angular velocity.

To calculate $\tilde{\mathbf{F}}_{O_{b}}^{i}$ and $\tilde{\mathbf{M}}_{O_{b}}^{i}$, the following equations are given by 


$$
\begin{aligned}
& \mathbf{v}_{O_{b}}^{1}=u \mathbf{e}_{\mathbf{x}}^{\mathbf{b}}+(w-b q) \mathbf{e}_{\mathbf{z}}^{\mathbf{b}}, \\
& \mathbf{v}_{O_{b}}^{2}=\left(u-\frac{l_{1}}{2} \Lambda^{\prime} \cos \Lambda\right) \mathbf{e}_{\mathbf{x}}^{\mathbf{b}}+\frac{l_{1}}{2} \Lambda^{\prime} \sin \Lambda \mathbf{e}_{\mathbf{y}}^{\mathbf{b}} \\
& +\left(w+\frac{l_{1}}{2} q \sin \Lambda\right) \mathbf{e}_{\mathbf{z}}^{\mathbf{b}} \\
& \mathbf{v}_{O_{b}}^{3}=\left(u-\Delta^{\prime} \sin \Lambda-l_{\Delta} \Lambda^{\prime} \cos \Lambda\right) \mathbf{e}_{\mathbf{x}}^{\mathbf{b}} \\
& +\left(l_{\Delta} \Lambda^{\prime} \sin \Lambda-\Delta^{\prime} \cos \Lambda\right) \mathbf{e}_{\mathbf{y}}^{\mathbf{b}}+\left(w+q l_{\Delta} \sin \Lambda\right) \mathbf{e}_{\mathbf{z}}^{\mathbf{b}}, \\
& \mathbf{v}_{O_{b}}^{4}=\left(u-\frac{l_{1}}{2} \Lambda^{\prime} \cos \Lambda\right) \mathbf{e}_{\mathbf{x}}^{\mathbf{b}}-\frac{l_{1}}{2} \Lambda^{\prime} \sin \Lambda \mathbf{e}_{\mathbf{y}}^{\mathbf{b}} \\
& +\left(w+\frac{l_{1}}{2} q \sin \Lambda\right) \mathbf{e}_{\mathrm{z}}^{\mathbf{b}} \\
& \mathbf{v}_{O_{b}}^{5}=\left(u-\Delta^{\prime} \sin \Lambda-l_{\Delta} \Lambda^{\prime} \cos \Lambda\right) \mathbf{e}_{\mathbf{x}}^{\mathbf{b}} \\
& +\left(\Delta^{\prime} \cos \Lambda-l_{\Delta} \Lambda^{\prime} \sin \Lambda\right) \mathbf{e}_{\mathbf{y}}^{\mathbf{b}}+\left(w+q l_{\Delta} \sin \Lambda\right) \mathbf{e}_{\mathbf{z}}^{\mathbf{b}}, \\
& \omega_{O_{b}}^{1}=q \mathbf{e}_{\mathbf{y}}^{\mathbf{b}} \text {, } \\
& \omega_{O_{b}}^{2}=\omega_{O_{b}}^{3}=q \mathbf{e}_{\mathbf{y}}^{\mathbf{b}}-\dot{\Lambda} \mathbf{e}_{\mathbf{z}}^{\mathbf{b}} \text {, } \\
& \omega_{O_{b}}^{4}=\omega_{O_{b}}^{5}=q \mathbf{e}_{\mathbf{y}}^{\mathbf{b}}+\dot{\Lambda} \mathbf{e}_{\mathbf{z}}^{\mathbf{b}} \text {, } \\
& \mathbf{J}_{O_{b}}^{1}=\operatorname{diag}\left(\begin{array}{lll}
J_{x} & J_{y} & J_{z}
\end{array}\right) \text {, } \\
& \mathbf{J}_{O_{b}}^{i}=\left[\begin{array}{ccc}
J_{i} \cos ^{2} \Lambda & -J_{i} \sin \Lambda \cos \Lambda & 0 \\
-J_{i} \sin \Lambda \cos \Lambda & J_{i} \sin ^{2} \Lambda & 0 \\
0 & 0 & J_{i}
\end{array}\right], \quad i=2,3, \\
& \mathbf{J}_{O_{b}}^{i}=\left[\begin{array}{ccc}
J_{i} \cos ^{2} \Lambda & J_{i} \sin \Lambda \cos \Lambda & 0 \\
J_{i} \sin \Lambda \cos \Lambda & J_{i} \sin ^{2} \Lambda & 0 \\
0 & 0 & J_{i}
\end{array}\right], \quad i=4,5,
\end{aligned}
$$

where $l_{\Delta}=l_{1}+\Delta-l_{2} / 2$. By simplifying body 2 , body 3 , body 4 , and body 5 into the rods with an even distribution of the weight, we get $J_{2}=J_{4}=m_{1} l_{1}^{2} / 12$ and $J_{3}=J_{5}=m_{2} l_{2}^{2} / 12$.

\section{B. Detailed Expressions of Kane Equations $f_{1}$, $f_{2}$, and $f_{3}$}

$$
\begin{aligned}
f_{1}= & (T+L \sin \alpha-D \cos \alpha)-m_{t} g \sin \theta-m_{t}(\dot{u}+q w) \\
& +m_{b} b q^{2}-m_{1} l_{1}\left(2 \Lambda^{\prime 2} \sin \Lambda-\Lambda^{\prime \prime} \cos \Lambda+q^{2} \sin \Lambda\right) \\
& -2 m_{2}\left(-3 \Delta^{\prime} \Lambda^{\prime} \cos \theta_{1}-\Delta^{\prime \prime} \sin \Lambda+2 l_{\Delta} \Lambda^{\prime 2} \sin \Lambda\right. \\
& \left.-l_{\Delta} \Lambda^{\prime \prime} \cos \Lambda+q^{2} l_{\Delta} \sin \Lambda\right), \\
f_{2}= & m_{t} g \cos \theta-D \sin \alpha-L \cos \alpha-m_{t} \dot{w} \\
& +m_{t} q u+m_{b} b \dot{q}-m_{1} l_{1}(2 q \dot{\Lambda} \cos \Lambda+\dot{q} \sin \Lambda) \\
& -m_{2}\left(2 l_{\Delta} \dot{q} \sin \Lambda+4 q \Delta^{\prime} \sin \Lambda+4 q l_{\Delta} \Lambda^{\prime} \cos \Lambda\right) \\
f_{3}= & -b\left(m_{b} g \cos \theta-D \sin \alpha-L \cos \alpha\right)+l_{1} m_{1} g \cos \theta \sin \Lambda \\
& +2 l_{\Delta} m_{2} g \cos \theta \sin \Lambda+M_{y}+m_{b} b(\dot{w}-b \dot{q}-q u) \\
& -l_{1} m_{1} \sin \Lambda\left(\dot{w}+q l_{1} \Lambda^{\prime} \cos \Lambda+\frac{l_{1}}{2} \dot{q} \sin \Lambda-q u\right) \\
& -2 m_{2} l_{\Delta} \sin \Lambda\left(\dot{w}+2 q \Delta^{\prime} \sin \Lambda+2 q l_{\Delta} \Lambda^{\prime} \cos \Lambda\right. \\
& \left.+\dot{q} l_{\Delta} \sin \Lambda-q u\right)-J_{y} \dot{q}-2\left(J_{2}+J_{3}\right) \\
& +\left(q \Lambda^{\prime} \sin \Lambda \cos \Lambda+\dot{q} \sin ^{2} \Lambda\right) .
\end{aligned}
$$

\section{Explicit Expressions of Full Longitudinal Dynamic Equation (9)}

$$
\begin{aligned}
& F_{1}=T \cos \alpha-D-m_{t} g \sin (\theta-\alpha)-q^{2} \cos \alpha\left(m_{1} l_{1} \sin \Lambda+2 m_{2} l_{\Delta} \sin \Lambda-m_{b} b\right) \\
& F_{2}=-\sin \alpha T-L+m_{t} g \cos (\theta-\alpha)+m_{t} q V+q^{2} \sin \alpha\left(m_{1} l_{1} \sin \Lambda+2 m_{2} l_{\Delta} \sin \Lambda-m_{b} b\right) \text {, } \\
& F_{3}=b \sin \alpha D+b \cos \alpha L+M_{y}+g \cos \theta\left(l_{1} m_{1} \sin \Lambda+2 l_{\Delta} m_{2} \sin \Lambda-m_{b} b\right)+q V \cos \alpha\left(l_{1} m_{1} \sin \Lambda+2 m_{2} l_{\Delta} \sin \Lambda-m_{b} b\right) \text {, }
\end{aligned}
$$

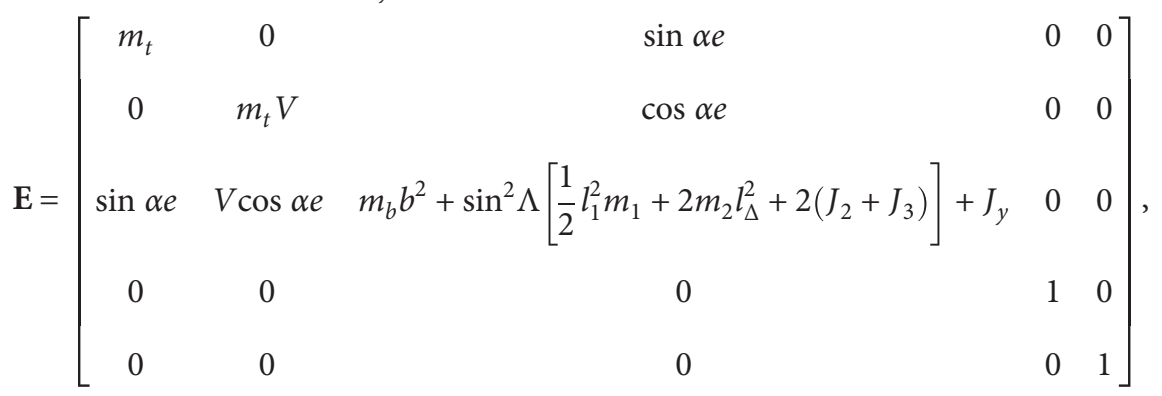




$$
\begin{aligned}
& W_{1}=-m_{1} l_{1} \cos \alpha\left(\Lambda^{\prime 2} \sin \Lambda-\Lambda^{\prime \prime} \cos \Lambda\right)-2 m_{2} \cos \alpha \\
& \cdot\left(-3 \Delta^{\prime} \Lambda^{\prime} \cos \Lambda-\Delta^{\prime \prime} \sin \Lambda+2 l_{\Delta} \Lambda^{\prime 2} \sin \Lambda-\Lambda^{\prime \prime} l_{\Delta} \cos \Lambda\right) \\
&-2 q m_{1} l_{1} \Lambda^{\prime \prime} \sin \alpha \cos \Lambda-m_{2} \sin \alpha \\
& \cdot\left(4 q \Delta^{\prime} \sin \Lambda+4 q l_{\Delta} \Lambda^{\prime} \cos \Lambda\right), \\
& W_{2}= m_{1} l_{1} \sin \alpha\left(\Lambda^{\prime 2} \sin \Lambda-\Lambda^{\prime \prime} \cos \Lambda\right)+2 m_{2} \sin \alpha \\
& \cdot\left(-3 \Delta^{\prime} \Lambda^{\prime} \cos \Lambda-\Delta^{\prime \prime} \sin \Lambda+2 l_{\Delta} \Lambda^{\prime 2} \sin \Lambda-\Lambda^{\prime \prime} l_{\Delta} \cos \Lambda\right) \\
&-2 q m_{1} l_{1} \Lambda^{\prime} \cos \alpha \cos \Lambda-m_{2} \cos \alpha \\
& \cdot\left(4 q \Delta^{\prime} \sin \Lambda+4 q l_{\Delta} \Lambda^{\prime} \cos \Lambda\right), \\
& W_{3}=-q m_{1} l_{1}^{2} \Lambda^{\prime} \sin \Lambda \cos \Lambda-2 m_{2} l_{\Delta} \sin \Lambda \\
& \cdot\left(2 q \Delta^{\prime} \sin \Lambda+2 q l_{\Delta} \Lambda^{\prime} \cos \Lambda\right) \\
&-2 q \Lambda^{\prime}\left(J_{2}+J_{3}\right) \sin \Lambda \cos \Lambda .
\end{aligned}
$$

\section{Aerodynamic Parameter Expressions}

$$
\begin{array}{rl}
C_{D}(\alpha)= & 0.0257-0.0069 \xi-0.0036 \lambda \\
& +(0.0014-0.0002 \xi-0.0008 \lambda) \alpha \\
C_{L_{\alpha=0}=} 0.1417 & +0.0642 \xi+0.0209 \lambda-0.0291 \xi^{2}-0.0336 \lambda \xi \\
-0.1527 \lambda^{2}+0.0159 \lambda \xi^{2}-0.0025 \lambda^{2} \xi+0.0565 \lambda^{3} \\
C_{L_{\alpha}=} & 0.0979+0.0051 \xi-0.0148 \lambda+0.0342 \lambda \xi \\
& -0.0632 \lambda^{2}-0.1548 \lambda^{2} \xi-0.1925 \lambda^{3} \\
& +0.1164 \lambda^{3} \xi+0.0992 \lambda^{4} \\
C_{L_{\delta e}} & 0.0038-0.0027 \xi+0.0012 \xi^{2} \\
C_{M_{\alpha=0}}= & 0.0357-0.0912 \xi-0.1026 \lambda+0.0702 \xi^{2} \\
& -0.2233 \lambda \xi+0.0674 \lambda^{2}-0.0264 \xi^{3} \\
& -0.0192 \lambda \xi^{2}+0.1672 \lambda^{2} \xi \\
C_{M_{\alpha}}= & -0.0293-0.0024 \xi-0.0245 \lambda+0.0083 \xi^{2} \\
& -0.0649 \lambda \xi-0.0758 \lambda^{2}-0.0932 \lambda \xi^{2} \\
& -0.1684 \lambda^{2} \xi+0.2344 \lambda^{3}-0.3587 \lambda^{2} \xi^{2} \\
& +0.1852 \lambda^{3} \xi-0.1849 \lambda^{4}-0.3052 \lambda^{3} \xi^{2} \\
& +0.0346 \lambda^{4} \xi+0.0401 \lambda^{5} \\
C_{M_{\delta e}} & =-0.0142+0.0083 \xi+0.0032 \lambda \\
& -0.0028 \xi^{2}-0.001 \lambda \xi \\
C_{M_{q}}= & -0.0323-0.001 \xi-0.0315 \lambda+0.0062 \xi^{2} \\
& -0.0521 \lambda \xi-0.0498 \lambda^{2}-0.0832 \lambda \xi^{2} \\
& -0.2184 \lambda^{2} \xi+0.1566 \lambda^{3}-0.4287 \lambda^{2} \xi^{2}
\end{array}
$$

\section{E. The Explicit Expressions of the Elements in} (12)

$$
\begin{aligned}
& A_{11}(\lambda, \xi)=-0.0229-0.0099 \xi+0.0032 \lambda+0.0042 \xi^{2} \\
& +0.0017 \lambda \xi-0.0012 \lambda^{2}, \\
& A_{12}(\lambda, \xi)=4.5878+2.1267 \xi+4.5612 \lambda-1.7067 \xi^{2} \\
& -2.7651 \lambda \xi-14.1742 \lambda^{2}-0.9305 \xi^{3} \\
& +1.8083 \lambda \xi^{2}+7.6959 \lambda^{2} \xi+28.2882 \lambda^{3} \\
& +0.1857 \xi^{4}-0.7726 \lambda \xi^{3}+5.8537 \lambda^{2} \xi^{2} \\
& -14.9300 \lambda^{3} \xi-30.1300 \lambda^{4}+0.1337 \xi^{5} \\
& -0.9328 \lambda \xi^{4}+1.9177 \lambda^{2} \xi^{3}-7.3449 \lambda^{3} \xi^{2} \\
& +10.4090 \lambda^{4} \xi+10.3441 \lambda^{5}, \\
& A_{22}(\lambda, \xi)=-2.5833-2.1035 \xi+0.3914 \lambda-0.0876 \xi^{2} \\
& -0.7024 \lambda \xi-1.6632 \lambda^{2}+0.0064 \xi^{3} \\
& -0.9041 \lambda \xi^{2}+2.7902 \lambda^{2} \xi+5.1029 \lambda^{3} \\
& -0.0012 \xi^{4}+0.0074 \lambda \xi^{3}+3.6357 \lambda^{2} \xi^{2} \\
& +0.8459 \lambda^{3} \xi-2.6484 \lambda^{4}+0.0021 \lambda \xi^{4} \\
& -0.0058 \lambda^{2} \xi^{3}-2.6835 \lambda^{3} \xi^{2}-1.9139 \lambda^{4} \xi, \\
& A_{32}(\lambda, \xi)=-24.3367-19.0056 \xi-4.8625 \lambda+13.6127 \xi^{2} \\
& -35.9235 \lambda \xi-91.0887 \lambda^{2}-0.2609 \xi^{3} \\
& -244.8501 \lambda \xi^{2}-229.3611 \lambda^{2} \xi+285.7218 \lambda^{3} \\
& -0.3695 \xi^{4}+45.0681 \lambda \xi^{3}+539.5873 \lambda^{2} \xi^{2} \\
& +214.2836 \lambda^{3} \xi-272.8778 \lambda^{4}-0.0185 \xi^{5} \\
& +1.2585 \lambda \xi^{4}-54.8806 \lambda^{2} \xi^{3}-353.7608 \lambda^{3} \xi^{2} \\
& +46.0297 \lambda^{4} \xi+80.4166 \lambda^{5} \\
& B_{11}(\lambda, \xi)=0.0191+0.0851 \xi-0.0283 \lambda-0.0369 \xi^{2} \\
& -0.1039 \lambda \xi-0.0258 \lambda^{2}+0.0177 \lambda \xi^{2} \\
& +0.0539 \lambda^{2} \xi+0.0099 \lambda^{3}, \\
& B_{21}(\lambda, \xi)=-0.1189-0.0057 \xi+0.0201 \lambda+0.0255 \xi^{2} \\
& +0.0077 \lambda \xi-0.0053 \lambda^{2}-0.0248 \xi^{3} \\
& -0.0009 \lambda \xi^{2}-0.0033 \lambda^{2} \xi, \\
& B_{31}(\lambda, \xi)=-14.3634-0.8088 \xi+4.3360 \lambda+2.2148 \xi^{2} \\
& +1.4978 \lambda \xi+0.6234 \lambda^{2}-1.5459 \xi^{3}-0.7150 \lambda \xi^{2} \\
& +0.7183 \lambda^{2} \xi-0.4237 \lambda^{3}-0.0803 \xi^{4}+0.2528 \lambda \xi^{3} \\
& +0.0444 \lambda^{2} \xi^{2}-0.5051 \lambda^{3} \xi \text {. }
\end{aligned}
$$

\section{Conflicts of Interest}

The authors declare that they have no conflicts of interest. 


\section{Acknowledgments}

This work was supported by the grants of the National Natural Science Foundation of China (no. 61563041 and no. 61305132) and Aeronautical Science Foundation of China (Grant no. 20135751040).

\section{References}

[1] T. A. Weisshaar, "Morphing aircraft systems: historical perspectives and future challenges," Journal of Aircraft, vol. 50, no. 2, pp. 337-353, 2013.

[2] T. Yue, L. Wang, and J. Ai, "Longitudinal linear parameter varying modeling and simulation of morphing aircraft," Journal of Aircraft, vol. 50, no. 6, pp. 1673-1681, 2013.

[3] J. Scarlett, R. Canfield, and B. Sanders, "Multibody dynamic aeroelastic simulation of a folding wing aircraft," in 47 th AIAA/ASME/ASCE/AHS/ASC Structures, Structural Dynamics, and Materials Conference, Newport, RI, USA, 2006.

[4] W. Jiang, C. Dong, and Q. Wang, "A systematic method of smooth switching LPV controllers design for a morphing aircraft," Chinese Journal of Aeronautics, vol. 28, no. 6, pp. 1640-1649, 2015.

[5] A. Marcos and G. J. Balas, "Development of linear-parametervarying models for aircraft," Journal of Guidance, Control, and Dynamics, vol. 27, no. 2, pp. 218-228, 2004.

[6] C. Hoffmann and H. Werner, "A survey of linear parametervarying control applications validated by experiments or high-fidelity simulations," IEEE Transactions on Control Systems Technology, vol. 23, no. 2, pp. 416-433, 2015.

[7] H. Alwi, C. Edwards, O. Stroosma, J. A. Mulder, and M. T. Hamayun, "Real-time implementation of an ISM faulttolerant control scheme for LPV plants," IEEE Transactions on Industrial Electronics, vol. 62, pp. 3896-3905, 2015.

[8] W. J. Rugh and J. S. Shamma, "Research on gain scheduling," Automatica, vol. 36, no. 10, pp. 1401-1425, 2000.

[9] H. Koroglu, "Observer-based linear parameter-varying control with guaranteed $L_{2}$-gain and $H_{2}$-type performance objectives," International Journal of Robust and Nonlinear Control, vol. 24, no. 14, pp. 2000-2017, 2014.

[10] P. Apkarian and R. J. Adams, "Advanced gain-scheduling techniques for uncertain systems," IEEE Transactions on Control Systems Technology, vol. 6, no. 1, pp. 21-32, 1998.

[11] F. Wu and K. Dong, "Gain-scheduling control of LFT systems using parameter-dependent Lyapunov functions," Automatica, vol. 42, no. 1, pp. 39-50, 2006.

[12] P. Apkarian, P. Gahinet, and G. Becker, "Self-scheduled $\mathrm{H}_{\infty}$ control of linear parameter-varying systems: a design example," Automatica, vol. 31, no. 9, pp. 1251-1261, 1995.

[13] C. Hoffmann, S. M. Hashemi, H. S. Abbas, and H. Werner, "Synthesis of LPV controllers with low implementation complexity based on a reduced parameter set," IEEE Transactions on Control Systems Technology, vol. 22, no. 6, pp. 2393-2398, 2014.

[14] P. Baranyi, “TP model transformation as a way to LMI-based controller design," IEEE Transactions on Industrial Electronics, vol. 51, no. 2, pp. 387-400, 2004.

[15] D. Peaucelle and D. Arzelier, "Robust performance analysis with LMI-based methods for real parametric uncertainty via parameter-dependent Lyapunov functions," IEEE Transactions on Automatic Control, vol. 46, no. 4, pp. 624-630, 2001.
[16] J. Guo, G. Tao, and Y. Liu, "A multivariable MRAC scheme with application to a nonlinear aircraft model," Automatica, vol. 47, no. 4, pp. 804-812, 2011.

[17] T. Yue, L. Wang, and J. Ai, "Gain self-scheduled $H_{\infty}$ control for morphing aircraft in the wing transition process based on an LPV model," Chinese Journal of Aeronautics, vol. 26, no. 4, pp. 909-917, 2013.

[18] R. Nazari, M. M. Seron, and J. A. De Dona, “Actuator fault tolerant control of systems with polytopic uncertainties using set-based diagnosis and virtual-actuator-based reconfiguration," Automatica, vol. 75, pp. 182-190, 2017.

[19] M. Sato and D. Peaucelle, "Gain-scheduled output-feedback controllers using inexact scheduling parameters for continuous-time LPV systems," Automatica, vol. 49, no. 4, pp. 1019-1025, 2013.

[20] Z. Li, X. Zhao, and J. Yu, "On robust control of continuoustime systems with state-dependent uncertainties and its application to mechanical systems," ISA Transactions, vol. 60, pp. 12-20, 2016.

[21] J.-L. Chang, "Dynamic output integral sliding-mode control with disturbance attenuation," IEEE Transactions on Automatic Control, vol. 54, no. 11, pp. 2653-2658, 2009.

[22] H. H. Choi, "Variable structure control of dynamical systems with mismatched norm-bounded uncertainties: an LMI approach," International Journal of Control, vol. 74, no. 13, pp. 1324-1334, 2001.

[23] J. C. Juang and C. M. Lee, "Design of sliding mode controllers with bounded $L_{2}$ gain performance: an LMI approach," International Journal of Control, vol. 78, no. 9, pp. 647-661, 2005.

[24] X. Hu, L. Wu, C. Hu, and H. Gao, "Adaptive sliding mode tracking control for a flexible air-breathing hypersonic vehicle," Journal of the Franklin Institute, vol. 349, no. 2, pp. 559-577, 2012.

[25] H. Alwi, C. Edwards, and A. Marcos, "Fault reconstruction using a LPV sliding mode observer for a class of LPV systems," Journal of the Franklin Institute, vol. 349, no. 2, pp. 510-530, 2012.

[26] D. Efimov, L. Fridman, T. Raissi, A. Zolghadri, and R. Seydou, "Interval estimation for LPV systems applying high order sliding mode techniques," Automatica, vol. 48, no. 9, pp. 23652371, 2012.

[27] S. Sivrioglu and K. Nonami, "Sliding mode control with timevarying hyperplane for AMB systems," IEEE/ASME Transactions on Mechatronics, vol. 3, no. 1, pp. 51-59, 1998.

[28] M. Tanelli, S. C. Strada, M. Corno, and A. Ferrara, "Sliding mode control for LPV systems," in American Control Conference (ACC), 2016, pp. 3686-3691, Boston, MA, USA, July 2016.

[29] X. Wei, H. Zhang, and L. Guo, "Composite disturbanceobserver-based control and terminal sliding mode control for uncertain structural systems," IFAC Proceedings Volumes, vol. 41, no. 2, pp. 5915-5920, 2008.

[30] J. Zhang and $\mathrm{S}$. Wu, "Dynamic modeling for a morphing aircraft and dynamic characteristics analysis," Journal of Beijing University of Aeronautics and Astronautics, vol. 41, pp. 58-64, 2015.

[31] S. Joshi, Z. Tidwell, W. Crossley, and S. Ramakrishnan, "Comparison of morphing wing strategies based upon aircraft performance impacts," in 45th AIAA/ASME/ASCE/ AHS/ASC Structures, Structural Dynamics \& Materials Conference, Palm Springs, CA, USA, 2004. 
[32] C. Yang, J. Han, S. Zheng, and O. Ogbobe Peter, "Dynamic modeling and computational efficiency analysis for a spatial 6-DOF parallel motion system," Nonlinear Dynamics, vol. 67, no. 2, pp. 1007-1022, 2012.

[33] Y. Wang, Y. Zhao, S. A. Bortoff, and K. Ueda, "A real-time energy-optimal trajectory generation method for a servomotor system," IEEE Transactions on Industrial Electronics, vol. 62, no. 2, pp. 1175-1188, 2015.

[34] T. H. Go and F. A. P. Lie, "Analysis of wing rock due to rollingmoment hysteresis," Journal of Guidance, Control, and Dynamics, vol. 31, no. 4, pp. 849-857, 2008.

[35] X. Su and Y. Jia, "Self-scheduled robust decoupling control with $\mathrm{H}_{\infty}$ performance of hypersonic vehicles," Systems and Control Letters, vol. 70, pp. 38-48, 2014.

[36] S. Nagy, Z. Petres, P. Baranyi, and H. Hashimoto, "Computational relaxed TP model transformation: restricting the computation to subspaces of the dynamic model," Asian Journal of Control, vol. 11, no. 5, pp. 461-475, 2009.

[37] Y. M. Jia, “Alternative proofs for improved LMI representations for the analysis and the design of continuous-time systems with polytopic type uncertainty: a predictive approach," IEEE Transactions on Automatic Control, vol. 48, pp. 14131416, 2003. 


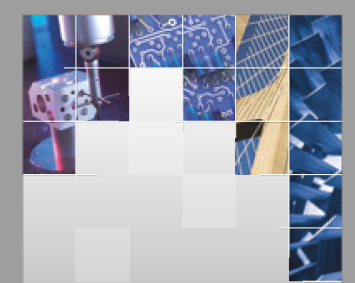

\section{Enfincering}
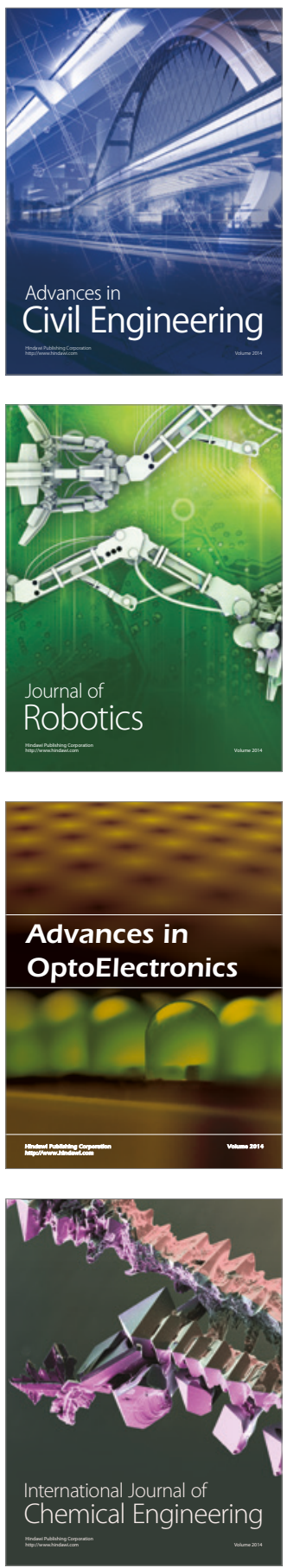

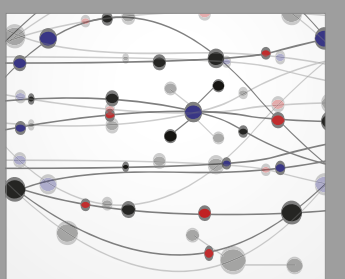

The Scientific World Journal

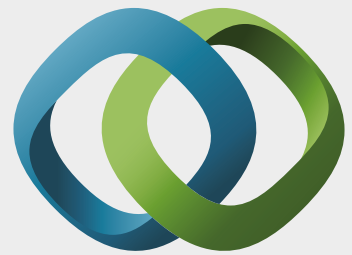

\section{Hindawi}

Submit your manuscripts at

https://www.hindawi.com
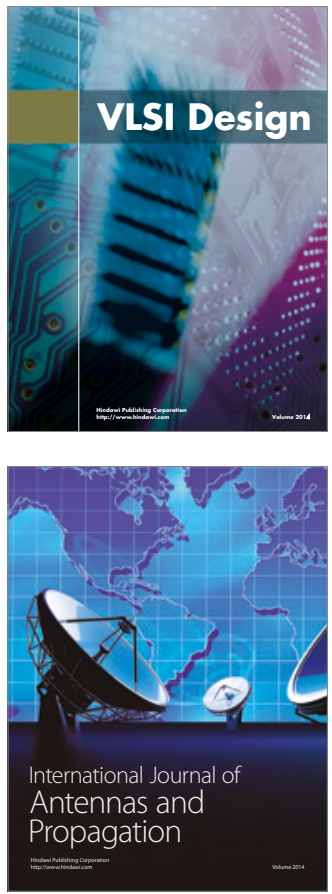

\section{Rotating}

Machinery
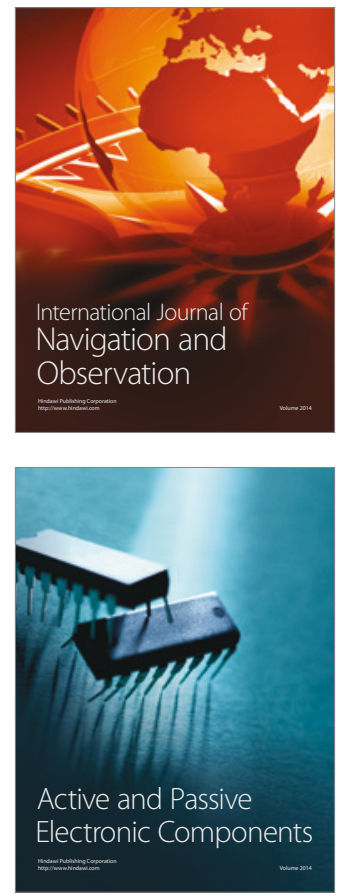
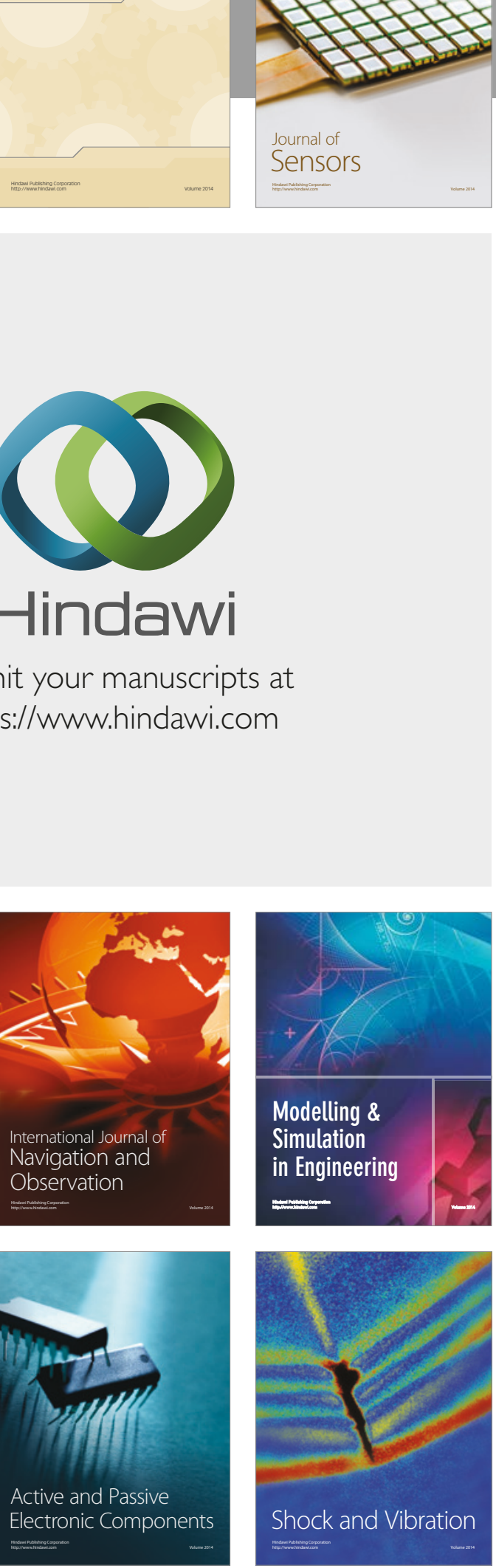
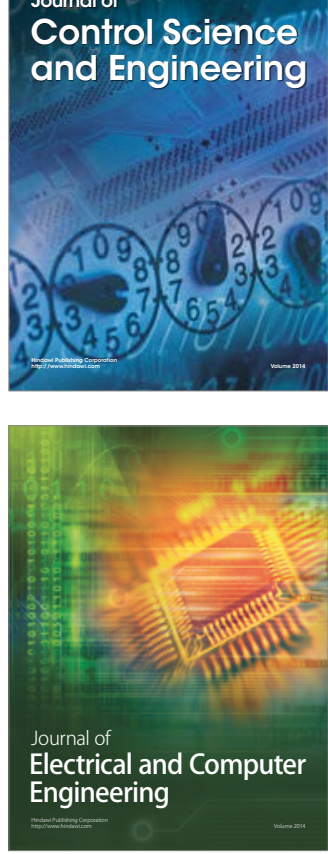

Distributed

Journal of

Control Science

and Engineering
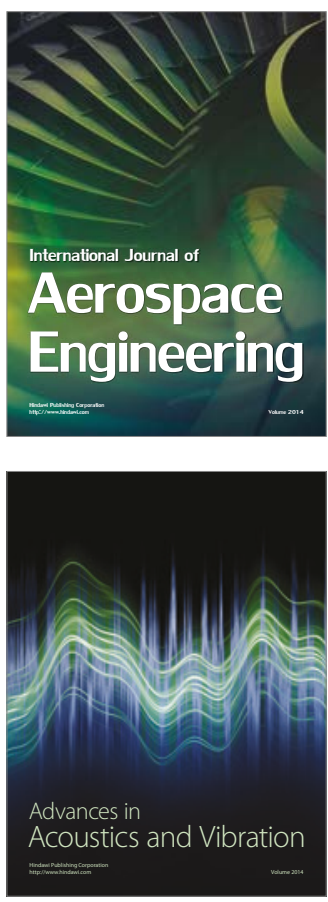

Sensor Networks 